\title{
Magneto-vortical effect in strong magnetic field
}

\section{Shu Lin and Lixin Yang}

School of Physics and Astronomy, Sun Yat-Sen University, 2 Daxue Rd, Tangjia, Zhuhai 519082, China

E-mail: linshu8@mail.sysu.edu.cn, yanglx5@mail2.sysu.edu.cn

ABSTRACT: We develop covariant chiral kinetic theory with Landau level basis. We use it to investigate a magnetized plasma with a transverse electric field and a steady vorticity as perturbations. After taking into account vacuum shift in the latter case, we find the resulting current and stress tensor in both cases can be matched consistently with constitutive equations of magnetohydrodynamics. We find the solution in the vorticity case contains both shifts in temperature and chemical potential as well as excitations of the lowest Landau level states. The solution gives rise to an vector charge density and axial current density. The vacuum parts coming from both shifts and excitations agree with previous studies and the medium parts coming entirely from excitations leads to a new contribution to vector charge and axial current density consistent with standard chiral vortical effect.

Keywords: Quark-Gluon Plasma, Anomalies in Field and String Theories, Chiral Lagrangians

ArXiv EPrint: 2103.11577 


\section{Contents}

1 Introduction 1

2 Covariant chiral kinetic theory with Landau level basis 3

3 Magnetized plasma with a drift $\quad 5$

3.1 Solution of CKT for a drift state 6

3.2 Matching with magnetohydrodynamics 8

4 Magnetized plasma with a vorticity $\quad 9$

4.1 Vortical solution 9

$\begin{array}{ll}\text { 4.2 Matching with magnetohydrodynamics } & 13\end{array}$

$\begin{array}{lll}4.3 \text { Vacuum ambiguity } & 15\end{array}$

$\begin{array}{llr}5 & \text { Summary } & 17\end{array}$

$\begin{array}{lr}\text { A Projection } & 18\end{array}$

$\begin{array}{ll}\text { B Momenta calculus } & 19\end{array}$

C Useful formulas $\quad 23$

\section{Introduction}

The response of QCD matter to magnetic field and vorticity has received much attention recently. In the linear regime, the response is the celebrated chiral magnetic effect (CME) [1-6] and chiral vortical effect (CVE) [5-10], which are known to be dictated by chiral anomaly and gravitational anomaly. While magnetic field and rotation are analogous in many ways, they differ in one crucial aspect. The magnetic field is external, but rotation is defined by motion of medium itself.

Recently the combined effect of magnetic field and vorticity has been studied by different groups [11-17]. In particular, it has been proposed by Hattori and Yin that in the limit of strong magnetic field, where lowest Landau level (LLL) approximation is valid, the effect of vorticity is to shift the energy of the LLL states through spin-orbit coupling [11]

$$
\Delta \epsilon^{ \pm}=\mp \frac{1}{2} \operatorname{sgn}\left(q_{f}\right) \hat{\mathbf{B}} \cdot \boldsymbol{\omega},
$$

where the upper and lower signs correspond to particle and anti-particle of both chiralities and $q_{f}$ is the charge of particle. The energy shift can also be interpreted as a shift of 
chemical potential $\operatorname{sgn}\left(q_{f}\right) \hat{\mathbf{B}} \cdot \boldsymbol{\omega}$ for particle. The shift induces vector charge density and axial current as

$$
\Delta J_{V}^{0}=q_{f} \frac{1}{4 \pi^{2}} \mathbf{B} \cdot \boldsymbol{\omega}, \quad \Delta \mathbf{J}_{A}=\left|q_{f}\right| \frac{1}{4 \pi^{2}}(\mathbf{B} \cdot \boldsymbol{\omega}) \hat{\mathbf{B}} .
$$

As remarked before, vorticity also implies circular motion of fluid velocity, which arises from average velocity of constituents in fluid cells. The rotation modifies the distribution of constituents in the plane transverse to the vorticity. It induces an extra contribution to (1.2). We will refer to this contribution as medium contribution, and (1.2) as vacuum contribution based on their different origins.

Indeed, medium contributions to (1.2) are expected. On the one hand, it is known that vector charge density receives the following contribution [18]

$$
\Delta J_{V}^{0}=-\nabla \cdot \mathbf{P}-2 \mathbf{M} \cdot \boldsymbol{\omega}
$$

The first term is the familiar bound charge from polarization $\mathbf{P}$, which is absent in fluid. The second term is a required relativistic counterpart of the first one. It is from the coupling of magnetization $\mathbf{M}$ and vorticity. On the other hand, if we view $\Delta \mathbf{J}_{A}$ in (1.2) as response to vorticity, we would expect also the standard CVE

$$
\Delta \mathbf{J}_{A}=\left(\frac{\mu^{2}+\mu_{5}^{2}}{2 \pi^{2}}+\frac{T^{2}}{6}\right) \boldsymbol{\omega} .
$$

We will confirm the medium contributions in (1.3) and (1.4) in a magnetized quantum electrodynamics plasma with a vorticity. The combined vacuum and medium contributions can be matched nicely with constitutive equation of magnetohydrodynamics (MHD) [1922] (see also [23-25]). We also study a closely related setting in which magnetized plasma is subject to transverse electric field. We will find the matching with MHD in this setting gives the same coefficients once the shift of chemical potential is carefully taken into account. In line with (1.2), we will work in the limit of strong magnetic field and use LLL approximation. The constituents of the fluid is LLL states and is described by chiral kinetic theory (CKT) with Landau level basis [26-30]. It is supplementary to the usual chiral kinetic theory with free fermion basis, which is best suited at weak external field [31-56].

This paper is organized as follows. In section 2, we derive the CKT with Landau level basis in a covariant form. In the presence of strong magnetic field and vorticity in the fluid, the CKT needs to be corrected by the second order gradient terms on gauge potential. In section 3, we find solution for magnetized plasma subject to transverse electric field. The resulting drift correction to the current and stress tensor are matched with MHD. We then follow a similar procedure to obtain the solution for magnetized plasma with a vorticity and compare it with MHD in section 4. We summarize and discuss possible extensions in section 5 .

Throughout this paper, we set $\hbar=1$ and $c=1$. We take positive charge $q_{f}=e$ for chiral fermions and absorb electric charge $e$ into the gauge field. We use the notations $\left(x^{\mu}\right)=\left(x_{0}, \mathbf{x}\right),\left(p^{\mu}\right)=\left(p_{0}, \mathbf{p}\right)$ for four-vectors and adopt mostly minus signature. 


\section{Covariant chiral kinetic theory with Landau level basis}

We start with a system of right-handed chiral fermions covariantly coupled to external gauge field. The two-point correlator $\bar{W}(z, y) \equiv\left\langle\psi(z) \psi(y)^{\dagger}\right\rangle$ satisfies the following equations

$$
\not D_{z} \bar{W}(z, y)=0, \quad \bar{W}(z, y) \not D_{y}^{\dagger}=0
$$

with the covariant derivatives defined as

$$
\not D_{z}=\not_{z}+i \not A(z), \quad \not D_{y}^{\dagger}=\overleftarrow{\not}_{y}-i \not A(y)
$$

where, for right-handed fermions, the slash is given by $A=\sigma^{\mu} A_{\mu}$. Note $\bar{W}(z, y)$ is not gauge invariant. A gauge invariant correlator $\tilde{W}(z, y)$ is constructed by using a gauge link $U(y, z)$ as

$$
\tilde{W}(z, y) \equiv \bar{W}(z, y) U(y, z)
$$

with the gauge link defined by

$$
U(y, z)=\exp \left(i \int_{z}^{y} d r^{\mu} A_{\mu}(r)\right) .
$$

In terms of $\tilde{W}(z, y)$, the EOM reads

$$
\begin{aligned}
& \not D_{z} \bar{W}(z, y)=D_{\mu}^{z}\left(\sigma^{\mu} \tilde{W}(z, y) U(z, y)\right)=0 \\
& \bar{W}(z, y) \not D_{y}^{\dagger}=\left(\tilde{W}(z, y) U(z, y) \sigma^{\mu}\right) D_{\mu}^{y^{\dagger}}=0
\end{aligned}
$$

It is convenient to switch to variables $x=\frac{1}{2}(y+z)$ and $s=y-z$. We will consider $x$ as a slow-varying variable and $s$ as a fast variable conjugate to momentum, i.e. $\partial_{x} \ll \partial_{s}$. This allows us to further simplify (2.5) using an expansion in $\partial_{x}$, which for the covariant derivatives and gauge link reads

$$
\begin{aligned}
D_{\mu}^{z} & =\frac{1}{2} \partial_{\mu}^{x}+\partial_{\mu}^{s}+i A_{\mu}(x)+\frac{i}{2}\left(s^{\nu} \partial_{\nu}^{x}\right) A_{\mu}(x)+\frac{i}{8}\left(s^{\nu} \partial_{\nu}^{x}\right)^{2} A_{\mu}(x)+O\left(\left(\partial_{x}\right)^{3}\right) \\
D_{\mu}^{y \dagger} & =\frac{1}{2} \overleftarrow{\partial}_{\mu}^{x}-\overleftarrow{\partial}_{\mu}^{s}-i A_{\mu}(x)+\frac{i}{2}\left(s^{\nu} \partial_{\nu}^{x}\right) A_{\mu}(x)-\frac{i}{8}\left(s^{\nu} \partial_{\nu}^{x}\right)^{2} A_{\mu}(x)+O\left(\left(\partial_{x}\right)^{3}\right) \\
U(y, z) & =\exp \left(i s^{\mu} A_{\mu}(x)+\frac{i s^{\mu}}{24}\left(s^{\nu} \partial_{\nu}^{x}\right)^{2} A_{\mu}(x)\right)+O\left(\left(\partial_{x}\right)^{4}\right)
\end{aligned}
$$

Commuting the covariant derivatives with the gauge link using the following identities

$$
\begin{aligned}
& D_{\mu}^{z}\left(U(z, y) \sigma^{\mu} \tilde{W}(z, y)\right) \\
& \quad=U(z, y)\left(\frac{1}{2} \partial_{\mu}^{x}-\partial_{\mu}^{s}+\frac{i}{2} s^{\nu} F_{\mu \nu}+\frac{i}{12}\left(s^{\lambda} \partial_{\lambda}^{x}\right) s^{\nu} F_{\mu \nu}\right)\left(\sigma^{\mu} \tilde{W}(x, s)\right) \\
& \left(\tilde{W}(z, y) \sigma^{\mu} U(z, y)\right) D_{\mu}^{y \dagger} \\
& \quad=\left(\tilde{W}(x, s) \sigma^{\mu}\right)\left(\frac{1}{2} \overleftarrow{\partial}_{\mu}^{x}+\overleftarrow{\partial}_{\mu}^{s}+\frac{i}{2} s^{\nu} F_{\mu \nu}-\frac{i}{12}\left(s^{\lambda} \partial_{\lambda}^{x}\right) s^{\nu} F_{\mu \nu}\right) U(z, y)
\end{aligned}
$$


we arrive at the EOM for $\tilde{W}(x, s)$ :

$$
\begin{aligned}
& \left(\frac{1}{2} \partial_{\mu}^{x}-\partial_{\mu}^{s}+\frac{i}{2} s^{\nu} F_{\mu \nu}+\frac{i}{12}\left(s^{\lambda} \partial_{\lambda}^{x}\right) s^{\nu} F_{\mu \nu}\right) \sigma^{\mu} \tilde{W}(x, s)=0, \\
& \left(\frac{1}{2} \partial_{\mu}^{x}+\partial_{\mu}^{s}+\frac{i}{2} s^{\nu} F_{\mu \nu}-\frac{i}{12}\left(s^{\lambda} \partial_{\lambda}^{x}\right) s^{\nu} F_{\mu \nu}\right) \tilde{W}(x, s) \sigma^{\mu}=0 .
\end{aligned}
$$

The kinetic equation is formulated with a quantum distribution function derivable from the Wigner transform of $\tilde{W}(x, s)$ [57-60]: $W(x, p)=\int \frac{d^{4} s}{(2 \pi)^{4}} e^{-i p \cdot s} \tilde{W}(x, s)$, which satisfies the following EOM

$$
\begin{aligned}
& \left(\frac{1}{2} \Delta_{\mu}-i \Pi_{\mu}\right) \sigma^{\mu} W(x, p)=0 \\
& \left(\frac{1}{2} \Delta_{\mu}+i \Pi_{\mu}\right) W(x, p) \sigma^{\mu}=0
\end{aligned}
$$

where we have defined operators $\Delta_{\mu}=\partial_{\mu}-\frac{\partial}{\partial p_{\nu}} F_{\mu \nu}, \Pi_{\mu}=p_{\mu}-\frac{1}{12} \frac{\partial^{2}}{\partial p_{\nu} \partial p_{\lambda}} \frac{\partial}{\partial x^{\lambda}} F_{\mu \nu}$ with the gradient $\frac{\partial}{\partial x^{\lambda}}$ acting on $F_{\mu \nu}$ only. We can rewrite (2.9) into component form by projecting it onto a suitable basis. For right-handed Weyl fermion, $W(x, p)$ is decomposed as

$$
W(x, p)=\frac{1}{2} j_{\mu} \bar{\sigma}^{\mu} .
$$

The projection of (2.9) gives the following EOM for components

$$
\begin{aligned}
\Pi_{\mu} j^{\mu} & =0, \\
\Delta_{\mu} j^{\mu} & =0, \\
\Pi^{\mu} j^{\nu}-\Pi^{\nu} j^{\mu} & =-\frac{1}{2} \epsilon^{\mu \nu \rho \sigma} \Delta_{\rho} j_{\sigma} .
\end{aligned}
$$

The details of the projection as well as the case for left-handed fermions can be found in appendix A. By solving the above equations, we can then obtain the current density and stress tensor by momentum integration of $j^{\mu}$ :

$$
\begin{aligned}
J^{\mu} & =\int d^{4} p \operatorname{tr}\left(\sigma^{\mu} W\right)=\int d^{4} p j^{\mu}, \\
T^{\mu \nu} & =\frac{1}{2} \int d^{4} p \operatorname{tr}\left(p^{\{\mu} \sigma^{\nu\}} W\right)=\frac{1}{2} \int d^{4} p p^{\{\mu} j^{\nu\}},
\end{aligned}
$$

where $X^{\{\mu} Y^{\nu\}} \equiv X^{\mu} Y^{\nu}+X^{\nu} Y^{\mu}$. The contribution of left-handed fermions will be added upon integrating over momenta.

Up to now, we have not specified the order of $F_{\mu \nu}$ in gradient. We can decompose $F_{\mu \nu}$ using the fluid velocity as: $F_{\mu \nu}=\epsilon_{\mu \nu \rho \sigma} u^{\rho} B^{\sigma}+E_{\mu} u_{\nu}-E_{\nu} u_{\mu}$, with $E^{\mu}$ and $B^{\mu}$ being electric and magnetic fields in local rest frame (LRF) of the fluid. For the case of our interest, we consider a strong background magnetic field and a possible electric field perturbation. Thus we regard $B^{\mu} \sim O\left(\partial^{0}\right)$ and $E^{\mu} \sim O(\partial)$. It follows that the $F_{\mu \nu}$ term in $\Pi_{\mu}$ can be $O\left(\partial^{0}\right)$. This is the reason to include the second term in $\Pi_{\mu}$, which counts as $O\left(\partial^{2}\right)$ on the gauge potential.

To proceed, we further choose a constant magnetic field in LRF of the fluid, $B^{\mu}=B b^{\mu}$ with $B$ and $b^{\mu}$ being magnitude and unit vector both constants in spacetime. While this 
choice is not the most general situation, it allows us to study the magneto-vortical effect in this simple setting. In particular, it adopts a simple covariant zeroth order solution as $[26,27]$

$$
j_{(0)}^{\mu}=(u+b)^{\mu} \delta(p \cdot(u+b)) f(p \cdot u) e^{\frac{p_{T}^{2}}{B}} \equiv(u+b)^{\mu} j
$$

where $p_{T}$ is the momentum component transverse to $u$ and $b$. It is defined by the transverse projector $P^{\mu \nu} \equiv-g^{\mu \nu}+u^{\mu} u^{\nu}-b^{\mu} b^{\nu}$ as $p_{T}^{\mu} \equiv-P^{\mu \nu} p_{\nu}=p^{\mu}-(p \cdot u) u^{\mu}+(p \cdot b) b^{\mu}$. The distribution function involving energy of fermion is

$$
f(p \cdot u)=\frac{2}{(2 \pi)^{3}} \sum_{r= \pm} \frac{r \theta(r p \cdot u)}{e^{r\left(p \cdot u-\mu_{R}\right) / T}+1},
$$

where $\mu_{R}$ is the chemical potential for right-handed Weyl fermions. We take constant chemical potential $\mu_{R / L}$ and temperature $T$ for simplicity. We note the background solution (2.13) and all the consequent perturbative solutions in the following sections are for the LLL. When the magnetic field is not strong enough, summation over Landau levels is needed. It is shown in [61] that, in the weak magnetic field limit, the summation $j^{\mu}$ will be reduced to the well-known solution proportional to momentum $p^{\mu}$.

In the next two sections, we will study first order gradient correction to (2.13) induced by constant transverse electric field and vorticity respectively. The resulting current and stress tensor allow us to study (thermal) Hall effect and magneto-vortical effect respectively. The static solution can also be matched with magnetohydrostatics, which is the static limit of magnetohydrodynamics. We will determine several thermodynamic functions through the matching.

\section{Magnetized plasma with a drift}

In this section, we study the magnetized plasma perturbed by a transverse electric field. This would lead to the development a drift velocity like in the case of magnetized plasma consisting of free fermions. We will see drift velocity appear in the solution. In this drift state, we will find the existence of charge and heat flow in the direction of the drift velocity. We start by turning on a perturbation $a^{\mu}$ at $O(1)$ in gauge potential which gives an $O(\partial)$ electric field $E_{\mu}=f_{\mu \nu} u^{\nu}$ in the transverse direction, i.e., $E_{\mu} b^{\mu}=0$. Here we have isolated the $O(\partial)$ field strength $f_{\mu \nu}=E_{\mu} u_{\nu}-E_{\nu} u_{\mu}$ from the $O(1)$ part $F_{\mu \nu}=\epsilon_{\mu \nu \rho \sigma} u^{\rho} B^{\sigma}$. It is sufficient to consider constant $u^{\mu}$, which allows us to drop gradient terms. The EOM are then modified to

$$
\begin{aligned}
& p_{\mu} j_{(1) \mathcal{D}}^{\mu}=0, \\
& D_{\mu} j_{(1) \mathcal{D}}^{\mu}-f_{\mu \nu} \frac{\partial}{\partial p_{\nu}} j_{(0)}^{\mu}=0, \\
& p^{[\mu} j_{(1) \mathcal{D}}^{\nu]}=-\frac{1}{2} \epsilon^{\mu \nu \rho \sigma}\left(D_{\rho} j_{\sigma}^{(1) \mathcal{D}}-f_{\rho \lambda} \frac{\partial}{\partial p_{\lambda}} j_{\sigma}^{(0)}\right),
\end{aligned}
$$

with $D_{\mu} \equiv-\frac{\partial}{\partial p_{\nu}} F_{\mu \nu}=\epsilon_{\mu \nu \rho \sigma} B b^{\rho} u^{\sigma} \frac{\partial}{\partial p_{\nu}}$ and $X^{[\mu} Y^{\nu]} \equiv X^{\mu} Y^{\nu}-X^{\nu} Y^{\mu}$. Below we will solve (3.1) and match the resulting current and stress tensor with MHD. 


\subsection{Solution of CKT for a drift state}

We start by putting down an ansatz at $O(\partial)$ for the drift state solution,

$$
j_{(1) \mathcal{D}}^{\mu}=(u+b)^{\mu} p \cdot u_{(1)}\left(\frac{\partial}{\partial(p \cdot u)} G_{1}+G_{2}\right)+u_{(1)}^{\mu} G_{3},
$$

with $u_{(1)}^{\mu} \equiv \frac{1}{2 B} \epsilon^{\mu \nu \rho \sigma} f_{\nu \rho} b_{\sigma}$. It is orthogonal to $E_{\mu}, b_{\mu}$ and $u_{\mu}$, and is interpreted as drift velocity. $G_{n} \propto \delta(p \cdot(u+b)) e^{\frac{p_{T}^{2}}{B}}$ are undetermined functions depending on momenta $p \cdot u, p \cdot b$ and $p_{T}^{2}$. We treat $p \cdot u, p \cdot b$ and $p_{T}^{2}$ as independent in momenta derivatives. More careful discussions can be found in appendix B where we take momentum in LRF of fluid $q^{\mu}$ as independent variables. ${ }^{1}$

One may think there might be a possible change in the leading order distribution $j_{(0)}^{\mu}$ due to the $O(1)$ perturbation $a^{\mu}$ in gauge potential,

$$
j_{(0) \mathcal{D}}^{\mu}=(u+b)^{\mu}\left(j+a^{\lambda} u_{\lambda} G_{4}\right) .
$$

Note that $a^{\lambda} u_{\lambda}$ can be interpreted as a shift on the chemical potential. Such a contribution is possible, but does not lead to charge/heat current in the direction of the drift velocity. We will not consider this possibility below. Now we work on the response to the external field $E^{\mu}$ at the first order. The two scalar equations (3.1a) and (3.1b) give

$$
\begin{gathered}
(3.1 \mathrm{a}) \rightarrow \quad p \cdot u_{(1)} p \cdot(u+b)\left(\frac{\partial}{\partial(p \cdot u)} G_{1}+G_{2}\right)+p \cdot u_{(1)} G_{3}=0, \\
(3.1 \mathrm{~b}) \rightarrow \quad-F_{\mu \lambda} \frac{\partial}{\partial p_{\lambda}} j_{(1) \mathcal{D}}^{\mu}-f_{\mu \nu} \frac{\partial}{\partial p_{\nu}} j_{(0)}^{\mu} \\
=B \epsilon_{\mu \lambda \alpha \beta} b^{\alpha} u^{\beta} \frac{\epsilon^{\mu \nu \rho \sigma} f_{\nu \rho} b_{\sigma}}{2 B} \frac{2 p_{T}^{\lambda}}{B} G_{3}-(u+b)^{\mu} f_{\mu \nu} \frac{2 p_{T}^{\nu}}{B} j \\
=p_{T}^{[\rho} u^{\nu]} f_{\nu \rho} \frac{1}{B} G_{3}-p_{T}^{[\nu} u^{\mu]} f_{\mu \nu} \frac{1}{B} j=0,
\end{gathered}
$$

where we have used $E^{\mu} u_{\mu}=0$ and $b^{\mu} f_{\mu \nu}=0$ in (3.5). One finds (3.4) and (3.5) are satisfied by $G_{1}=G_{3}=j$ and $G_{2} \propto \delta(p \cdot(u+b))$.

We simplify the anti-symmetric tensor equation (3.1c) as follows. For the left hand side (l.h.s.), we have

$$
\begin{aligned}
p^{[\mu} j_{(1) \mathcal{D}}^{\nu]}= & p \cdot u_{(1)} p^{[\mu}(u+b)^{\nu]}\left(\frac{\partial}{\partial(p \cdot u)} G_{1}+G_{2}\right)+p^{[\mu} u_{(1)}^{\nu]} G_{3} \\
= & p \cdot u_{(1)}\left(p_{T}^{[\mu}(u+b)^{\nu]}-p \cdot(u+b) b^{[\mu} u^{\nu]}\right)\left(\frac{\partial}{\partial(p \cdot u)} G_{1}+G_{2}\right) \\
& +\left(p_{T}^{[\mu} u_{(1)}^{\nu]}+p \cdot u(u+b)^{[\mu} u_{(1)}^{\nu]}\right) G_{3},
\end{aligned}
$$

where we have used $p_{T}^{[\mu}(u+b)^{\nu]}=p^{[\mu}(u+b)^{\nu]}+p \cdot(u+b) b^{[\mu} u^{\nu]}$ in the first term and $p^{[\mu} u_{(1)}^{\nu]}=p_{T}^{[\mu} u_{(1)}^{\nu]}+p \cdot u(u+b)^{[\mu} u_{(1)}^{\nu]}$ by on shell condition in the second term. For two parts on the right hand side (r.h.s.),

$$
-\frac{1}{2} \epsilon^{\mu \nu \rho \sigma}\left(-F_{\rho \lambda} \frac{\partial}{\partial p_{\lambda}} j_{\sigma}^{(1) \mathcal{D}}-f_{\rho \lambda} \frac{\partial}{\partial p_{\lambda}} j_{\sigma}^{(0)}\right),
$$

\footnotetext{
${ }^{1}$ One can equivalently switch between $p^{\mu}$ and $q^{\mu}$ to arrive at the same final solution.
} 
the first one can be written as

$$
\begin{aligned}
& -\frac{1}{2} \epsilon^{\mu \nu \rho \sigma} B \epsilon_{\rho \lambda \alpha \beta} b^{\alpha} u^{\beta} \frac{\partial}{\partial p_{\lambda}}\left[p \cdot u_{(1)}(u+b)_{\sigma}\left(\frac{\partial}{\partial(p \cdot u)} G_{1}+G_{2}\right)+u_{\sigma}^{(1)} G_{3}\right] \\
& =\left(p \cdot u_{(1)} p_{T}^{[\mu}(u+b)^{\nu]}+\frac{B}{2} u_{(1)}^{[\mu}(u+b)^{\nu]}\right)\left(\frac{\partial}{\partial(p \cdot u)} G_{1}+G_{2}\right)+b^{[\mu} u^{\nu]} p \cdot u_{(1)} G_{3},
\end{aligned}
$$

where we have used $u \cdot u_{(1)}=b \cdot u_{(1)}=0$. Noting $f_{\rho \lambda} b^{\lambda}=0$, the second part writes

$$
\frac{1}{2} \epsilon^{\mu \nu \rho \sigma} f_{\rho \lambda}(u+b)_{\sigma}\left(u^{\lambda} \frac{\partial}{\partial(p \cdot u)}+\frac{2 p_{T}^{\lambda}}{B}\right) j=\frac{B}{2}(u+b)^{[\mu} u_{(1)}^{\nu]} \frac{\partial}{\partial(p \cdot u)} j+p_{T}^{[\mu} u_{(1)}^{\nu]} j,
$$

where we have used the following identities shown in appendix $\mathrm{C}$,

$$
\begin{aligned}
& \epsilon^{\mu \nu \rho \sigma} f_{\rho \lambda}(u+b)_{\sigma} u^{\lambda}=B(u+b)^{[\mu} u_{(1)}^{\nu]}, \\
& \epsilon^{\mu \nu \rho \sigma} f_{\rho \lambda}(u+b)_{\sigma} p_{T}^{\lambda}=B p_{T}^{[\mu} u_{(1)}^{\nu]} .
\end{aligned}
$$

We collect the l.h.s. and r.h.s. from (3.6)(3.8)(3.9) and group them into $b^{[\mu} u^{\nu]}, p_{T}^{[\mu}(u+$ $b)^{\nu]},(u+b)^{[\mu} u_{(1)}^{\nu]}$ and $p_{T}^{[\mu} u_{(1)}^{\nu]}$ terms to fix $G_{n}$ by comparing the coefficients of the groups. For $b^{[\mu} u^{\nu]}$ terms, one gets

$$
-p \cdot u_{(1)} p \cdot(u+b)\left(\frac{\partial}{\partial(p \cdot u)} G_{1}+G_{2}\right)=p \cdot u_{(1)} G_{3},
$$

which holds by $G_{1}=G_{3}=j$. The coefficients of $p_{T}^{[\mu}(u+b)^{\nu]}$ on two sides cancel out automatically. For the $(u+b)^{[\mu} u_{(1)}^{\nu]}$ terms, we get

$$
p \cdot u G_{3}=-\frac{B}{2}\left(\frac{\partial}{\partial(p \cdot u)} G_{1}+G_{2}\right)+\frac{B}{2} \frac{\partial}{\partial(p \cdot u)} j
$$

which, with $G_{1}=G_{3}=j$, gives $G_{2}=\frac{-2 p \cdot u}{B} j$. The coefficients of $p_{T}^{[\mu} u_{(1)}^{\nu]}$ give $G_{3}=j$. In summary, the full drift solution for right-handed fermions is

$$
j_{(1) \mathcal{D}}^{\mu}=p \cdot u_{(1)}(u+b)^{\mu}\left(\frac{\partial}{\partial(p \cdot u)}-\frac{2 p \cdot u}{B}\right) j+u_{(1)}^{\mu} j .
$$

In fact, up to $O(\partial)$ the solution can be combined with the zeroth order solution into a more suggestive form

$$
j_{(0)}^{\mu}+j_{(1) \mathcal{D}}^{\mu}=\left(u_{\mathcal{D}}+b\right)^{\mu} \delta\left(p \cdot\left(u_{\mathcal{D}}+b\right)\right) f\left(p \cdot u_{\mathcal{D}}\right) e^{\left(p^{2}-\left(p \cdot u_{\mathcal{D}}\right)^{2}+(p \cdot b)^{2}\right) / B} .
$$

This is nothing but the zeroth order solution with $u^{\mu} \rightarrow u_{\mathcal{D}}^{\mu} \equiv\left(u+u_{(1)}\right)^{\mu}$. The counterpart for left-handed fermions can be obtained by sending $b \rightarrow-b$ and $\mu_{R} \rightarrow \mu_{L}$.

We may either choose $u_{\mathcal{D}}^{\mu}$ or $u^{\mu}$ as fluid velocity, which correspond to different frame choices in hydrodynamics. In the former case the in medium electric field defined by $u_{\mathcal{D}}$ is vanishing $f_{\mu \nu} u^{\nu}+F_{\mu \nu} u_{(1)}^{\nu}=0$. It follows that there is no charge/heat current orthogonal to the fluid velocity. This corresponds to the Landau frame. The latter case contains both charge/heat current. As we will see below, it can be matched with the constitutive equations of MHD in thermodynamic frame [19]. 


\subsection{Matching with magnetohydrodynamics}

With (2.13) and (3.13), we are ready to calculate the current and stress tensor by momenta integration. Here we simply collect the final results and leave the details of the evaluation to appendix B.

$$
\begin{aligned}
J_{(0)}^{\mu} & =\frac{\mu B}{2 \pi^{2}} u^{\mu}+\frac{\mu_{5} B}{2 \pi^{2}} b^{\mu}, \\
T_{(0)}^{\mu \nu} & =\frac{\chi_{V} B}{2 \pi^{2}}\left(u^{\mu} u^{\nu}+b^{\mu} b^{\nu}\right)+\frac{\chi_{A}}{2 \pi^{2}} u^{\{\mu} b^{\nu\}}, \\
J_{(1) \mathcal{D}}^{\mu} & =-\frac{\mu}{2 \pi^{2}} \epsilon^{\mu \nu \rho \sigma} u_{\nu} E_{\rho} b_{\sigma}, \\
T_{(1) \mathcal{D}}^{\mu \nu} & =-\frac{1}{2 \pi^{2}}\left(\frac{B}{4}+\chi\right) u^{\{\mu} \epsilon^{\nu\} \lambda \rho \sigma} u_{\lambda} E_{\rho} b_{\sigma},
\end{aligned}
$$

where we have defined $\chi_{V} \equiv \frac{\mu^{2}+\mu_{5}^{2}}{2}+\frac{\pi^{2} T^{2}}{6}$ and $\chi_{A} \equiv \mu \mu_{5}$. We see that (3.15a) contains charge density and current density contributions. From the charge density, we easily recognize the charge susceptibility $\chi_{\mu}=\frac{B}{2 \pi^{2}}$, which is given by density of LLL states. The current density is the celebrated CME result. Eq. (3.15b) is effectively reduced to $1+1$ dimensional in the LLL approximation and there is net longitudinal heat flow in the presence of $\mu_{5}$. As we stressed the in medium electric field $E_{\mu}=F_{\mu \nu} u^{\nu}$ in thermodynamic frame leads to Hall current and heat current in (3.15c) and (3.15d).

To match with constitutive equations of MHD, which relates components of current and stress tensor through thermodynamic functions, we closely follow the notations of [19], in which the current and stress tensor are decomposed $a^{2}$

$$
\begin{aligned}
J^{\mu} & =\mathcal{N} u^{\mu}+\mathcal{J}^{\mu}, \\
T^{\mu \nu} & =\mathcal{E} u^{\mu} u^{\nu}+\mathcal{P} \Delta^{\mu \nu}+\mathcal{Q}^{\mu} u^{\nu}+\mathcal{Q}^{\nu} u^{\mu}+\mathcal{T}^{\mu \nu},
\end{aligned}
$$

where $\Delta^{\mu \nu} \equiv-g^{\mu \nu}+u^{\mu} u^{\nu}=P^{\mu \nu}+b^{\mu} b^{\nu}$. One has $\mathcal{N}=u_{\mu} J^{\mu}, \mathcal{J}_{\mu}=-\Delta_{\mu \lambda} J^{\lambda}, \mathcal{E}=u_{\mu} u_{\nu} T^{\mu \nu}$, $\mathcal{P}=\frac{1}{3} \Delta_{\mu \nu} T^{\mu \nu}, \mathcal{Q}_{\mu}=-\Delta_{\mu \alpha} u_{\beta} T^{\alpha \beta}$ and $\mathcal{T}^{\mu \nu}=\frac{1}{2}\left(\Delta_{\mu \alpha} \Delta_{\nu \beta}+\Delta_{\nu \alpha} \Delta_{\mu \beta}-\frac{2}{3} \Delta_{\mu \nu} \Delta_{\alpha \beta}\right) T^{\alpha \beta}$. From (3.15a) through (3.15d), we obtain the components of the current and stress tensor at $O(1)$,

$$
\begin{aligned}
\mathcal{N}_{(0)}=\frac{\mu B}{2 \pi^{2}}, & \mathcal{J}_{(0)}^{\mu}=\frac{\mu_{5} B}{2 \pi^{2}} b^{\mu}, \\
\mathcal{E}_{(0)}=\frac{\chi_{V} B}{2 \pi^{2}}, & \mathcal{Q}_{(0)}^{\mu}=\frac{\chi_{A} B}{2 \pi^{2}} b^{\mu}, \\
\mathcal{P}_{(0)}=\frac{\chi_{V} B}{6 \pi^{2}}, & \mathcal{T}_{(0)}^{\mu \nu}=\frac{\chi_{V} B}{6 \pi^{2}}\left(2 b^{\mu} b^{\nu}-P^{\mu \nu}\right),
\end{aligned}
$$

and two nonvanishing parity odd components at $O(\partial)$,

$$
\begin{aligned}
\mathcal{J}_{(1) \mathcal{D}}^{\mu} & =-\frac{\mu}{2 \pi^{2}} \epsilon^{\mu \nu \rho \sigma} u_{\nu} E_{\rho} b_{\sigma}, \\
\mathcal{Q}_{(1) \mathcal{D}}^{\mu} & =-\frac{1}{2 \pi^{2}}\left(\frac{B}{4}+\chi\right) \epsilon^{\mu \nu \rho \sigma} u_{\nu} E_{\rho} b_{\sigma} .
\end{aligned}
$$

\footnotetext{
${ }^{2}$ In making the comparison, we note that [19] uses a different signature in metric. Also their definition of electromagnetic field or alternatively current differs from ours by a sign. We quote the converted constitutive relations of MHD.
} 
The spatial current $\mathcal{J}_{(1) \mathcal{D}}^{\mu}$ along the drift velocity gives the Hall conductivity $\sigma_{H}=\frac{\mu}{2 \pi^{2}}$. The heat flow $\mathcal{Q}_{(1) \mathcal{D}}^{\mu}$ is parallel to the Hall current. They are nonvanishing in the absence of $\mu_{5}$.

Meanwhile, the constitutive relations for components of MHD [19] give,

$$
\begin{array}{ll}
\mathcal{N}_{(0)}=n=p_{, \mu}, & \mathcal{P}_{(0)}=\Pi=p-\frac{4}{3} p_{, B^{2}} B^{2} \\
\mathcal{E}_{(0)}=\epsilon=-p+T p_{, T}+\mu p_{, \mu}, & \mathcal{T}_{(0)}^{\mu \nu}=\frac{1}{3} \alpha_{B B} B^{2}\left(2 b^{\mu} b^{\nu}-P^{\mu \nu}\right),
\end{array}
$$

and

$$
\begin{aligned}
& \mathcal{J}_{(1)}^{\mu}=-\alpha_{B B}, \mu \\
& \epsilon^{\mu \nu \rho \sigma} u_{\nu} E_{\rho} B_{\sigma}, \\
& \mathcal{Q}_{(1)}^{\mu}=\left(M_{\omega, \mu}+2 p_{, B^{2}}\right) \epsilon^{\mu \nu \rho \sigma} u_{\nu} E_{\rho} B_{\sigma},
\end{aligned}
$$

where $p$ is pressure, $\alpha_{B B}=2 p_{, B^{2}}$ is magnetic susceptibility and $M_{\omega}$ is magneto-vortical susceptibility. Note that $p$ and $\Pi$ are thermodynamic functions here, not to be confused with indexed $p_{\mu}$ and $\Pi_{\mu}$. To compare with MHD, we mute $\mu_{5}$ to get $\chi_{V} \rightarrow \chi \equiv \frac{\mu^{2}}{2}+$ $\frac{\pi^{2} T^{2}}{6}$ and vanishing parity odd coefficient $\chi_{A}$. Then one easily finds the $O(1)$ components in (3.17a) satisfy the constitutive relations in (3.18a) by taking $p=\chi B / 2 \pi^{2}$. At $O(\partial)$, by matching $(3.17 \mathrm{~b})$ and $(3.18 \mathrm{~b})$, we can fix $M_{\omega}$ in drift state as

$$
M_{\omega}^{\mathcal{D}}=-\frac{\mu}{8 \pi^{2}}-\frac{\xi}{2 \pi^{2} B} .
$$

\section{Magnetized plasma with a vorticity}

In this section, we study the effects of a steady vorticity parallel to the magnetic field in the plasma. We turn on a vorticity $\omega^{\mu}=\frac{1}{2} \epsilon^{\mu \nu \rho \sigma} u_{\nu} \partial_{\rho} u_{\sigma}=\omega b^{\mu}$ in the fluid along the direction of the magnetic field with $\omega=-\omega^{\mu} b_{\mu}$. We further require the absence of shear or bulk tensors in the fluid. Then we solve (2.11) to the first order of gradient, or equivalently, $O(\omega)$. The solution is to be referred to as vortical solution. In matching the resulting current and stress tensor with MHD, we find one of the thermodynamic functions $M_{\omega}$ has a different value from (3.19). The apparent discrepancy will be resolved with a reinterpretation of the results, which precisely corresponds to shift of chemical potential discussed in the introduction.

\subsection{Vortical solution}

Denoting the first order solution by $j_{(1) \mathcal{V}}^{\mu}$, we can write the equations explicitly as

$$
\begin{aligned}
p_{\mu} j_{(1) \mathcal{V}}^{\mu}+\delta \Pi_{\mu} j_{(0)}^{\mu} & =0 \\
\partial_{\mu} j_{(0)}^{\mu}+D_{\mu} j_{(1) \mathcal{V}}^{\mu} & =0 \\
p^{[\mu} j_{(1) \mathcal{V}}^{\nu]}+\delta \Pi^{[\mu} j_{(0)}^{\nu]} & =-\frac{1}{2} \epsilon^{\mu \nu \rho \sigma}\left(\partial_{\rho} j_{\sigma}^{(0)}+D_{\rho} j_{\sigma}^{(1) \mathcal{V}}\right),
\end{aligned}
$$

where we have defined $\delta \Pi_{\mu} \equiv \Pi_{\mu}-p_{\mu}$. Here we choose $D_{\mu}=\epsilon_{\mu \nu \rho \sigma} B b^{\rho} u^{\sigma} \frac{\partial}{\partial p_{\nu}}$ corresponding to a constant magnetic field in the LRF of the fluid. The field strength $F_{\mu \nu}=\epsilon_{\mu \nu \rho \sigma} B b^{\rho} u^{\sigma}$ 
is spacetime dependent through the fluid velocity. In $(4.1), j_{(1) \mathcal{V}}^{\mu}$ is sourced by terms proportional to $\partial j_{(0)}$ and $\delta \Pi j_{(0)}$. The former captures the spacetime derivatives on the distribution function and the latter is the counterpart in field strength. While mathematically they both reduce to spacetime dependence of fluid velocity, their physical difference is clear. Accordingly we will split $j_{(1) \mathcal{V}}^{\mu}$ into two parts

$$
j_{(1) \mathcal{V}}^{\mu}=j_{(1) \mathcal{C}}^{\mu}+j_{(1) \mathcal{A}}^{\mu}
$$

with $j_{(1) \mathcal{C}}^{\mu}$ and $j_{(1) \mathcal{A}}^{\mu}$ satisfying (4.3) and (4.4) respectively.

$$
\begin{aligned}
p_{\mu} j_{(1) \mathcal{C}}^{\mu} & =0 \\
\partial_{\mu} j_{(0)}^{\mu}+D_{\mu} j_{(1) \mathcal{C}}^{\mu} & =0 \\
p^{[\mu} j_{(1) \mathcal{C}}^{\nu]} & =-\frac{1}{2} \epsilon^{\mu \nu \rho \sigma}\left(\partial_{\rho} j_{\sigma}^{(0)}+D_{\rho} j_{\sigma}^{(1) \mathcal{C}}\right), \\
p_{\mu} j_{(1) \mathcal{A}}^{\mu}+\delta \Pi_{\mu} j_{(0)}^{\mu} & =0 \\
D_{\mu} j_{(1) \mathcal{A}}^{\mu} & =0 \\
p^{[\mu} j_{(1) \mathcal{A}}^{\nu]}+\delta \Pi^{[\mu} j_{(0)}^{\nu]} & =-\frac{1}{2} \epsilon^{\mu \nu \rho \sigma}\left(D_{\rho} j_{\sigma}^{(1) \mathcal{A}}\right) .
\end{aligned}
$$

Similar to (3.2), we take the following ansatz for $j_{(1) \mathcal{C}}^{\mu}$,

$$
j_{(1) \mathcal{C}}^{\mu}=(u+b)^{\mu} \frac{\omega p \cdot p_{T}}{B}\left(\frac{\partial}{\partial(p \cdot u)} F_{1}+F_{2}\right)+\frac{\omega p_{T}^{\mu}}{B} F_{3}+(u+b)^{\mu} F_{4},
$$

where $F_{n} \propto \delta(p \cdot(u+b)) e^{\frac{p_{T}^{2}}{B}}$ are undetermined functions. Note that the ansatz is a Lorentz vector by construction, thus independent of an artificial frame vector. This is in contrast to the conventional approaches [62,63], where the frame dependence of distribution function at $O(\partial)$ is canceled by the frame dependence of the spin tensor contribution. Our ansatz may be viewed as a combined contribution of the two. The same is also true for the ansatz in (4.13).

Noting the on shell condition $\delta(p \cdot(u+b))$ in $F_{n},(4.3 a)$ gives

$$
\begin{aligned}
p_{\mu} j_{(1) \mathcal{C}}^{\mu} & =p \cdot(u+b)\left(\frac{\omega p_{T}^{2}}{B}\left(\frac{\partial}{\partial(p \cdot u)} F_{1}+F_{2}\right)+F_{4}\right)+p_{\mu} \frac{\omega p_{T}^{\mu}}{B} F_{3} \\
& =\frac{\omega}{B}\left(-p_{T}^{2} F_{1}+p_{T}^{2} F_{3}\right)=0, \\
\rightarrow \quad & -F_{1}+F_{3}=0,
\end{aligned}
$$

where we have used integration by parts for the $\frac{\partial}{\partial(p \cdot u)} F_{1}$ term and $p_{\mu} p_{T}^{\mu}=p_{T}^{2}$.

We turn to $(4.3 \mathrm{~b})$, which can be simplified using the bulk free condition $\partial_{\mu} u^{\mu}=0$. In this case, $(4.3 \mathrm{~b})$ becomes

$$
\begin{aligned}
& \partial_{\mu} j_{(0)}^{\mu}-D_{\mu} j_{(1) \mathcal{C}}^{\mu}=(u+b)^{\mu} p^{\lambda} \partial_{\mu} u_{\lambda}\left(\frac{\partial}{\partial(p \cdot u)}-\frac{2 p \cdot u}{B}\right) j \\
& \quad+B \epsilon_{\mu \nu \rho \sigma} b^{\rho} u^{\sigma} \frac{\partial}{\partial p_{\nu}}\left[(u+b)^{\mu}\left(\frac{\omega p_{T}^{2}}{B}\left(\frac{\partial}{\partial(p \cdot u)} F_{1}+F_{2}\right)+F_{4}\right)+\frac{\omega p_{T}^{\mu}}{B} F_{3}\right]=0 .
\end{aligned}
$$


Furthermore, with $\partial_{\rho} u_{\sigma}=-\omega b^{\mu} u^{\nu} \epsilon_{\mu \nu \rho \sigma}$ following from the shear free condition, one finds all the terms vanish by anti-symmetry of $\epsilon_{\mu \nu \rho \sigma}$. Therefore (4.3b) is automatically satisfied.

The anti-symmetric tensor equation requires some work. Firstly, we simplify the l.h.s. and r.h.s. of (4.3c) as follows. The l.h.s. writes

$$
\begin{aligned}
p^{[\mu} j_{(1) \mathcal{C}}^{\nu]}= & p^{[\mu}(u+b)^{\nu]}\left(\frac{\omega p_{T}^{2}}{B}\left(\frac{\partial}{\partial(p \cdot u)} F_{1}+F_{2}\right)+F_{4}\right)+\frac{\omega}{B} p^{[\mu} p_{T}^{\nu]} F_{3} \\
= & \left(p_{T}^{[\mu}(u+b)^{\nu]}-p \cdot(u+b) b^{[\mu} u^{\nu]}\right)\left(\frac{\omega p_{T}^{2}}{B}\left(\frac{\partial}{\partial(p \cdot u)} F_{1}+F_{2}\right)+F_{4}\right) \\
& -\frac{\omega}{B} p \cdot u p_{T}^{[\mu}(u+b)^{\nu]} F_{1},
\end{aligned}
$$

where we have used $p_{T}^{[\mu}(u+b)^{\nu]}=p^{[\mu}(u+b)^{\nu]}+p \cdot(u+b) b^{[\mu} u^{\nu]}$ in the first term and $p^{[\mu} p_{T}^{\nu]}=-p \cdot u p_{T}^{[\mu}(u+b)^{\nu]}$ by on shell condition in the second term. Eq. (4.8) contains two independent structures $p_{T}^{[\mu}(u+b)^{\nu]}$ and $b^{[\mu} u^{\nu]}$, which are transverse-longitudinal and longitudinal-temporal types. There are two parts on the r.h.s.,

$$
-\frac{1}{2} \epsilon^{\mu \nu \rho \sigma} \partial_{\rho} j_{\sigma}^{(0)}-\frac{1}{2} \epsilon^{\mu \nu \rho \sigma} D_{\rho} j_{\sigma}^{(1) \mathcal{C}} .
$$

Using the relation $\epsilon^{\mu \nu \rho \sigma} \partial_{\rho} u_{\sigma}=2 \omega\left(b^{\mu} u^{\nu}-b^{\nu} u^{\mu}\right)$ and $\partial_{\rho} u_{\lambda}=-\omega \epsilon_{\rho \lambda \alpha \beta} b^{\alpha} u^{\beta}$, we can simplify the first term as

$$
\begin{aligned}
& -\frac{1}{2} \epsilon^{\mu \nu \rho \sigma}\left[\partial_{\rho} u_{\sigma}+(u+b)_{\sigma} p^{\lambda} \partial_{\rho} u_{\lambda}\left(\frac{\partial}{\partial(p \cdot u)}-\frac{2 p \cdot u}{B}\right)\right] j \\
& =-\omega\left[b^{[\mu} u^{\nu]}-\frac{1}{2} \epsilon^{\mu \nu \rho \sigma}(u+b)_{\sigma} p^{\lambda} \epsilon_{\rho \lambda \alpha \beta} b^{\alpha} u^{\beta}\left(\frac{\partial}{\partial(p \cdot u)}-\frac{2 p \cdot u}{B}\right)\right] j \\
& =-\omega\left[b^{[\mu} u^{\nu]} j+\frac{1}{2} p_{T}^{[\mu}(u+b)^{\nu]}\left(\frac{\partial j}{\partial(p \cdot u)}-\frac{2 p \cdot u}{B} j\right)\right] .
\end{aligned}
$$

In the second part, given that $F_{n} \propto e^{\frac{p_{T}^{2}}{B}}$ depend on momenta by $p \cdot u, p \cdot b$ and $p_{T}^{2}$ only, we note when acting on $j_{\sigma}^{(1) \mathcal{C}}$, the operator $\frac{\partial}{\partial p_{\lambda}}$ can pull out terms like $u^{\lambda} \frac{\partial}{\partial(p \cdot u)}, b^{\lambda} \frac{\partial}{\partial p \cdot b}, p_{T}^{\lambda} \frac{\partial}{\partial p_{T}^{2}}$ and $\delta_{\sigma}^{\lambda}$, where only the last two cases survive upon contraction with $\epsilon_{\rho \lambda \alpha \beta}$. One gets

$$
\begin{gathered}
-\frac{1}{2} \epsilon^{\mu \nu \rho \sigma} \epsilon_{\rho \lambda \alpha \beta} B b^{\alpha} u^{\beta} \frac{\partial}{\partial p_{\lambda}}\left[(u+b)_{\sigma}\left(\frac{\omega p_{T}^{2}}{B}\left(\frac{\partial}{\partial(p \cdot u)} F_{1}+F_{2}\right)+F_{4}\right)+\frac{\omega p_{\sigma}^{T}}{B} F_{3}\right] \\
=-\frac{1}{2} \epsilon^{\mu \nu \rho \sigma} \epsilon_{\rho \lambda \alpha \beta} B b^{\alpha} u^{\beta}\left[(u+b)_{\sigma} \frac{2 p_{T}^{\lambda}}{B}\left(\omega\left(1+\frac{p_{T}^{2}}{B}\right)\left(\frac{\partial}{\partial(p \cdot u)} F_{1}+F_{2}\right)+F_{4}\right)\right. \\
\left.+\frac{\omega}{B}\left(\delta_{\sigma}^{\lambda}+\frac{2 p_{T}^{\lambda} p_{\sigma}^{T}}{B}\right) F_{1}\right] \\
=p_{T}^{[\mu}(u+b)^{\nu]}\left(\omega\left(1+\frac{p_{T}^{2}}{B}\right)\left(\frac{\partial}{\partial(p \cdot u)} F_{1}+F_{2}\right)+F_{4}\right)+b^{[\mu} u^{\nu]} \omega\left(1+\frac{p_{T}^{2}}{B}\right) F_{1} .
\end{gathered}
$$

The r.h.s. from (4.10)(4.11) contains the same structures as the l.h.s. By matching the coefficient of $b^{[\mu} u^{\nu]}$ using integration by part, we can fix $F_{1}=j$. The remaining structure 
reads

$$
\text { 1.h.s. }- \text { r.h.s. }=-\omega p_{T}^{[\mu}(u+b)^{\nu]}\left[\frac{2 p \cdot u}{B} j+\left(\frac{1}{2} \frac{\partial}{\partial(p \cdot u)} j+F_{2}\right)\right] \text {. }
$$

We note that $F_{4}$ cancels in (4.12). In fact, $F_{4} \propto \delta(p \cdot(u+b)) e^{\frac{p_{T}^{2}}{B}}$, which can be recognized as the change of distribution function. We also note that $F_{2} \propto \delta(p \cdot(u+b))$ while $\frac{\partial}{\partial(p \cdot u)} j$ contains $\delta^{\prime}(p \cdot(u+b))$, which immediately shows (4.12) cannot be identically zero. This will be resolved only after we combine with the solution $j_{(1) \mathcal{A}}^{\mu}$.

To solve for $j_{(1) \mathcal{A}}^{\mu}$, we note that (4.4) can be formally obtained from the zeroth order by the replacement $p_{\mu} \rightarrow p_{\mu}+\delta \Pi_{\mu}$ and $j_{(0)}^{\mu} \rightarrow j_{(0)}^{\mu}+j_{(1) \mathcal{A}}^{\mu}$ and expanded to $O(\partial)$. The formal solution motivates the following ansatz

$$
j_{(1) \mathcal{A}}^{\mu}=(u+b)^{\mu} \delta^{\prime}(p \cdot(u+b))(u+b) \cdot \delta \Pi \tilde{j},
$$

where $\tilde{j} \equiv f(p \cdot u) e^{\frac{p_{T}^{2}}{B}} . \delta \Pi$ is a differential operator, whose explicit expression is worked out in appendix B as

$$
\begin{aligned}
& \delta \Pi_{\mu}=u_{\mu} \delta \Pi_{u}+\frac{2 p_{\mu}^{T}}{B} \delta \Pi_{T} \quad \text { with } \\
& \delta \Pi_{u}=\frac{B \omega}{12} P_{\lambda \nu} \frac{\partial}{\partial p_{\lambda}} \frac{\partial}{\partial p_{\nu}}, \quad \delta \Pi_{T}=\frac{B \omega}{12} \frac{\partial}{\partial(p \cdot u)},
\end{aligned}
$$

up to $O(\omega)$. Below we verify (4.13) gives an extra contribution that cancels out the $\frac{\partial}{\partial(p \cdot u)} j$ term in (4.12) and fixes $F_{2} \propto \delta(p \cdot(u+b))$ to gives a proper final solution at $O(\omega)$. Using $p_{T} \cdot u=p_{T} \cdot b=0,(4.4 \mathrm{a})$ gives

$$
\begin{aligned}
& p_{\mu} j_{(1) \mathcal{A}}^{\mu}+\left(u_{\mu} \delta \Pi_{u}+\frac{2 p_{\mu}^{T}}{B} \delta \Pi_{T}\right) j_{(0)}^{\mu} \\
& =p \cdot(u+b) \delta^{\prime}(p \cdot(u+b)) \delta \Pi_{u} \tilde{j}+\delta \Pi_{u}(\delta(p \cdot(u+b)) \tilde{j})=0 .
\end{aligned}
$$

Note that $\delta \Pi_{u}$ involves differentiation on the transverse momenta $p_{T}^{\mu}$ and therefore does not act on $p \cdot u, p \cdot b$, which means $\delta \Pi_{u} \delta(p \cdot(u+b))=\delta(p \cdot(u+b)) \delta \Pi_{u}$. We can then see the above equation holds upon integration by parts. By the anti-symmetric $\epsilon_{\mu \nu \rho \sigma} b^{\rho} u^{\sigma}$ term in $D_{\mu},(4.4 \mathrm{~b})$ is trivially satisfied as $j_{(1) \mathcal{A}}^{\mu} \propto(u+b)^{\mu}$. We then proceed to the anti-symmetric tensor equation (4.4c) as follows. Explicitly, the l.h.s. of (4.4c) writes

$$
\begin{aligned}
\delta \Pi^{[\mu} j_{(0)}^{\nu]}+p^{[\mu} j_{(1) \mathcal{A}}^{\nu]}= & \left(u^{[\mu} b^{\nu]} \delta \Pi_{u}+\frac{2}{B} p_{T}^{[\mu}(u+b)^{\nu]} \delta \Pi_{T}\right)(\delta(p \cdot(u+b)) \tilde{j}) \\
& +p^{\mu}(u+b)^{\nu}\left(\delta^{\prime}(p \cdot(u+b)) \delta \Pi_{u} \tilde{j}\right) \\
= & \left(\frac{2}{B} p_{T}^{[\mu}(u+b)^{\nu]} \delta \Pi_{T}-b^{[\mu} u^{\nu]} \delta \Pi_{u}\right)(\delta(p \cdot(u+b)) \tilde{j}) \\
& +\left(p_{T}^{\mu}(u+b)^{\nu}-p \cdot(u+b) b^{[\mu} u^{\nu]}\right)\left(\delta^{\prime}(p \cdot(u+b)) \delta \Pi_{u} \tilde{j}\right) \\
= & p_{T}^{[\mu}(u+b)^{\nu]}\left(\frac{2}{B} \delta \Pi_{T}(\delta(p \cdot(u+b)) \tilde{j})+\delta^{\prime}(p \cdot(u+b)) \delta \Pi_{u} \tilde{j}\right),
\end{aligned}
$$


where we have canceled out the $b^{[\mu} u^{\nu]}$ terms using integration by parts in the last equality. The r.h.s. of (4.4c) gives

$$
\begin{aligned}
-\frac{1}{2} \epsilon^{\mu \nu \rho \sigma} \frac{\partial}{\partial p_{\lambda}} \epsilon_{\rho \lambda \alpha \beta} B b^{\alpha} u^{\beta} j_{\sigma}^{(1) \mathcal{A}} & =-\frac{1}{2} \epsilon^{\mu \nu \rho \sigma} \epsilon_{\rho \lambda \alpha \beta} B b^{\alpha} u^{\beta}(u+b)_{\sigma} \delta^{\prime}(p \cdot(u+b)) \delta \Pi_{u} \frac{\partial}{\partial p_{\lambda}} \tilde{j} \\
& =p_{T}^{\mu}(u+b)^{\nu} \delta^{\prime}(p \cdot(u+b))\left(\delta \Pi_{u}-\frac{\omega}{3}\right) \tilde{j}
\end{aligned}
$$

where the $\frac{\omega}{3}$ term in the last equality comes from the commutator $\left[\delta \Pi_{u}, p_{T}^{\lambda}\right]$. Now, gathering (4.16) and (4.17), we have

$$
\begin{aligned}
\text { l.h.s. }- \text { r.h.s. } & =p_{T}^{[\mu}(u+b)^{\nu]}\left(\frac{2}{B} \delta \Pi_{T}(\delta(p \cdot(u+b)) \tilde{j})+\frac{\omega}{3} \delta^{\prime}(p \cdot(u+b)) \tilde{j}\right) \\
& =p_{T}^{[\mu}(u+b)^{\nu]}\left(\frac{\omega}{6} \delta(p \cdot(u+b)) f^{\prime}(p \cdot u) e^{\frac{p_{T}^{2}}{B}}+\frac{\omega}{2} \delta^{\prime}(p \cdot(u+b)) \tilde{j}\right) .
\end{aligned}
$$

One finds the cancellation of (4.12) and (4.18) indeed requires an on shell $F_{2}$,

$$
F_{2}=-\frac{1}{3} \delta(p \cdot(u+b)) f^{\prime}(p \cdot u) e^{\frac{p_{T}^{2}}{B}}-\frac{2 p \cdot u}{B} j .
$$

Combining (4.5) and (4.13), we have the following solution up to possible addition of $F_{4}$ as

$$
\begin{aligned}
j_{(1) \mathcal{V}}^{\mu}=( & +b)^{\mu}\left[-\frac{\omega}{3}\left(\frac{p_{T}^{2}}{B}+1\right) \delta^{\prime}(p \cdot(u+b)) f(p \cdot u)+\frac{2 \omega p_{T}^{2}}{3 B} \delta(p \cdot(u+b)) f^{\prime}(p \cdot u)\right. \\
& \left.-\frac{2 \omega p_{T}^{2}}{B^{2}} p \cdot u \delta(p \cdot(u+b)) f(p \cdot u)\right] e^{\frac{p_{T}^{2}}{B}}+\frac{\omega p_{T}^{\mu}}{B} \delta(p \cdot(u+b)) f(p \cdot u) e^{\frac{p_{T}^{2}}{B}}
\end{aligned}
$$

The above procedure can be easily generalized to the case of left-handed fermions with the solution given by the replacement $b \rightarrow-b$ and $\mu_{R} \rightarrow \mu_{L} \cdot{ }^{3}$

\subsection{Matching with magnetohydrodynamics}

Again, after integration over momenta and summation over right/left-handed contributions detailed in appendix B, (4.20) gives the current and stress tensor as

$$
\begin{aligned}
J_{(1) \mathcal{V}}^{\mu}= & \frac{\omega}{2 \pi^{2}}\left(2 \chi_{V}+\frac{2}{3} B\right) u^{\mu}+\frac{\omega}{2 \pi^{2}} 2 \chi_{A} b^{\mu} \\
T_{(1) \mathcal{V}}^{\mu \nu}= & \frac{\omega}{2 \pi^{2}}\left(2 \xi_{V}+\frac{2}{3} \mu B\right) u^{\mu} u^{\nu}+\frac{\omega}{2 \pi^{2}}\left(2 \xi_{V}-\frac{1}{3} \mu B\right) b^{\mu} b^{\nu} \\
& +\frac{\omega}{2 \pi^{2}}\left(2 \xi_{A}+\frac{1}{6} \mu_{5} B\right) u^{\{\mu} b^{\nu\}}+\frac{\omega}{2 \pi^{2}} \frac{\mu B}{2} P^{\mu \nu},
\end{aligned}
$$

where we have defined $\xi_{V} \equiv \frac{1}{3} \mu\left(\mu^{2}+3 \mu_{5}^{2}+\pi^{2} T^{2}\right)$ and $\xi_{A} \equiv \frac{1}{3} \mu_{5}\left(\mu_{5}^{2}+3 \mu^{2}+\pi^{2} T^{2}\right)$. We note that the current density in (4.21a) is in agreement with CVE. The charge density does contains an $O\left(B^{0}\right)$ medium contribution and $O(B)$ vacuum contribution. The latter however contradicts (1.2). The contradiction should not be a surprise. The reason is our

\footnotetext{
${ }^{3}$ Here it is more appropriate to regard $b$ as the spin direction of LLL states rather than the magnetic field direction.
} 
vortical solution (4.20) is unique only up to possible addition of $F_{4}$, which we have not considered so far. In fact, since we consider the magnetized plasma in a steady vorticity, the state is not reached as a response to vorticity, thus we do not have a first principle to fix $F_{4}$ within our approach. We can choose any $F_{4} \propto \delta(p \cdot(u+b)) e^{\frac{p_{T}^{2}}{B}} g(p \cdot u)$, which necessarily modifies $J_{(1) \mathcal{V}}^{\mu}$ and $T_{(1) \mathcal{V}}^{\mu \nu}$.

Fortunately the ambiguity can still be fixed by matching with constitutive equations of MHD. From (4.21a) and (4.21b), we obtain the components of the current and stress tensor at $O(\partial)$,

$$
\begin{array}{ll}
\mathcal{N}_{(1) \mathcal{V}}=\frac{\omega}{2 \pi^{2}}\left(2 \chi_{V}+\frac{2}{3} B\right), & \mathcal{J}_{(1) \mathcal{V}}^{\mu}=\frac{\omega}{2 \pi^{2}} 2 \chi_{A} b^{\mu} \\
\mathcal{E}_{(1) \mathcal{V}}=\frac{\omega}{2 \pi^{2}}\left(2 \xi_{V}+\frac{2}{3} \mu B\right), & \mathcal{Q}_{(1) \mathcal{V}}^{\mu}=\frac{\omega}{2 \pi^{2}}\left(2 \xi_{A}+\frac{1}{6} \mu_{5} B\right) b^{\mu} \\
\mathcal{P}_{(1) \mathcal{V}}=\frac{\omega}{6 \pi^{2}}\left(2 \xi_{V}+\frac{2}{3} \mu B\right), & \mathcal{T}_{(1) \mathcal{V}}^{\mu \nu}=\frac{\omega}{6 \pi^{2}}\left(2 \xi_{V}-\frac{5}{6} \mu B\right)\left(2 b^{\mu} b^{\nu}-P^{\mu \nu}\right),
\end{array}
$$

for which, the constitutive relations in MHD [19] are

$$
\begin{aligned}
\mathcal{N}_{(1)} & =f_{\mathcal{N}}=-2\left(2 p_{, B^{2}}+M_{\omega, \mu}\right) B \omega, \\
\mathcal{E}_{(1)} & =f_{\mathcal{E}}=-2\left(T M_{\omega, T}+\mu M_{\omega, \mu}-2 M_{\omega}\right) B \omega, \\
\mathcal{P}_{(1)} & =f_{\mathcal{P}}=\frac{2}{3}\left(M_{\omega}+4 M_{\omega, B^{2}} B^{2}\right) B \omega, \\
\mathcal{T}_{(1)} & =f_{\mathcal{T}}=-\frac{4}{3}\left(M_{\omega, B^{2}} B^{2}+M_{\omega}\right) B \omega,
\end{aligned}
$$

with $\mathcal{T}_{(1)}^{\mu \nu}=\left(2 b^{\mu} b^{\nu}-P^{\mu \nu}\right) \mathcal{T}_{(1)}$. To proceed, we turn off $\mu_{5}$ to get $\chi_{V} \rightarrow \chi \equiv \frac{\mu^{2}}{2}+\frac{\pi^{2} T^{2}}{6}, \xi_{V} \rightarrow$ $\xi \equiv \frac{1}{3} \mu\left(\mu^{2}+\pi^{2} T^{2}\right)$ and vanishing parity odd coefficients $\chi_{A}, \xi_{A}$. Then the counterparts in CKT are reduced to

$$
\begin{aligned}
& \mathcal{N}_{(1) \mathcal{V}}=f_{\mathcal{N}}^{\prime}=\frac{\omega}{2 \pi^{2}}\left(2 \chi+\frac{2}{3} B\right), \\
& \mathcal{E}_{(1) \mathcal{V}}=f_{\mathcal{E}}^{\prime}=\frac{\omega}{2 \pi^{2}}\left(2 \xi+\frac{2}{3} \mu B\right), \\
& \mathcal{P}_{(1) \mathcal{V}}=f_{\mathcal{P}}^{\prime}=\frac{\omega}{6 \pi^{2}}\left(2 \xi+\frac{2}{3} \mu B\right), \\
& \mathcal{T}_{(1) \mathcal{V}}=f_{\mathcal{T}}^{\prime}=\frac{\omega}{6 \pi^{2}}\left(2 \xi-\frac{5}{6} \mu B\right),
\end{aligned}
$$

up to possible addition of $F_{4}$, which corresponds to $O(\omega)$ modification of distribution. The simplest possible modification is through $O(\omega)$ modification of temperature and chemical potential. If this were the case, the effect of $F_{4}$ can be realized by a frame transformation, which amounts to a redefinition of temperature and chemical potential [64]. We will see below a frame transformation indeed allows for matching with MHD. The matching is most easily done through the following frame invariant variables [19],

$$
\begin{aligned}
f & \equiv f_{\mathcal{P}}-\left(\frac{\partial \Pi}{\partial \epsilon}\right)_{n} f_{\mathcal{E}}-\left(\frac{\partial \Pi}{\partial n}\right)_{\epsilon} f_{\mathcal{N}}, \\
t & \equiv f_{\mathcal{T}}-\frac{B^{2}}{3}\left[\left(\frac{\partial \alpha_{B B}}{\partial \epsilon}\right)_{n} f_{\mathcal{E}}+\left(\frac{\partial \alpha_{B B}}{\partial n}\right)_{\epsilon} f_{\mathcal{N}}\right] .
\end{aligned}
$$


We should match $f$ and $t$ constructed using (4.23) and (4.24) to fix $M_{\omega}$. Using

$$
\begin{aligned}
& \epsilon=\frac{\chi B}{2 \pi^{2}} \\
& \Pi=\frac{\chi B}{6 \pi^{2}}, \\
& n=\frac{\mu B}{2 \pi^{2}} \\
& \Rightarrow \quad\left(\frac{\partial \Pi}{\partial n}\right)_{\epsilon}=0, \quad\left(\frac{\partial \Pi}{\partial \epsilon}\right)_{n}=\frac{1}{3}, \quad\left(\frac{\partial \alpha_{B B}}{\partial n}\right)_{\epsilon}=0, \quad\left(\frac{\partial \alpha_{B B}}{\partial \epsilon}\right)_{n}=\frac{1}{B^{2}} \text {, }
\end{aligned}
$$

in (4.25), one gets

$$
\begin{aligned}
\frac{2}{3}\left(M_{\omega}+4 M_{\omega, B^{2}} B^{2}\right) & +\frac{2}{3}\left(T M_{\omega, T}+\mu M_{\omega, \mu}-2 M_{\omega}\right)=0 \\
\frac{4}{3}\left(M_{\omega, B^{2}} B^{2}+M_{\omega}\right)-\frac{2}{3}\left(T M_{\omega, T}+\mu M_{\omega, \mu}-2 M_{\omega}\right) & =\frac{\mu}{4 \pi^{2}}
\end{aligned}
$$

which are satisfied by $M_{\omega}^{\mathcal{V}}=\frac{\mu}{8 \pi^{2}}+\frac{\# \xi}{B}$. An arbitrary coefficient \# is allowed in the medium part. By matching the medium part with (3.19), we fix

$$
M_{\omega}^{\mathcal{V}}=\frac{\mu}{8 \pi^{2}}-\frac{\xi}{2 \pi^{2} B}
$$

We see the matching equations (4.27) are over-determined. The agreement on the medium part of $M_{\omega}$ between drift and vortical solutions is rather non-trivial. The disagreement on the vacuum part needs further clarification.

\subsection{Vacuum ambiguity}

Recall $M_{\omega}$ is defined by the change of free energy in response to magneto-vortical source [64], which reads in our case

$$
\Delta \mathcal{F}=-2 M_{\omega} B \omega
$$

The definition implicitly assumes the vacuum is not changed as the magneto-vortical source is turned on adiabatically. If the vacuum state is changed in the process, we should instead use the new vacuum state as reference point in calculating the free energy. To reconcile $M_{\omega}^{\mathcal{D}}$ and $M_{\omega}^{\mathcal{V}}$, the vacuum energy density needs to be lowered by $\frac{\mu B \omega}{2 \pi^{2}}$ in the adiabatic process above. Indeed this is consistent with the picture that each LLL state has a lowered energy (1.1). The chemical potential measured with respect to the lowered vacuum is shifted up by $\Delta \mu=\omega$ for particles. $\frac{\mu B \omega}{2 \pi^{2}}$ is accounted by the product of $\Delta \mu$ and charge density $\frac{\mu B}{2 \pi^{2}}$ of the LLL states.

The new vacuum is given by $j^{\mu}=j_{(0)}^{\mu}+j_{\text {vac }}^{\mu}$, with the shift part reads

$$
j_{\mathrm{vac}}^{\mu}=\omega(u \pm b)^{\mu} \delta(p \cdot(u \pm b)) f^{\prime}(p \cdot u) e^{\frac{p_{T}^{2}}{B}}
$$

with upper/lower signs for right/left handed fermions respectively. Eq. (4.30) has the simple interpretation as from a shift in chemical potential $\delta \mu_{\mathrm{vac}}=-\omega$. The corresponding shift in stress tensor and current are evaluated as

$$
T_{\text {vac }}^{\mu \nu}=\frac{1}{2} \int d^{4} p p^{\{\mu} j_{\text {vac }}^{\nu\}}=-\frac{\mu B \omega}{2 \pi^{2}}\left(u^{\mu} u^{\nu}+b^{\mu} b^{\nu}\right), \quad J_{\text {vac }}^{\mu}=\int d^{4} p j_{\text {vac }}^{\mu}=-\frac{B \omega}{2 \pi^{2}} u^{\mu} .
$$


Note that apart from the needed shift in energy density, there is also a negative shift of charge density in the new vacuum.

Now we can calculate the change of charge density using $M_{\omega}^{\mathcal{V}}$ and the vacuum shifted density (4.31) as

$$
\Delta J^{0}=-2\left(2 p_{, B^{2}}+M_{\omega, \mu}^{\mathcal{V}}\right) B \omega-J_{\text {vac }}^{0}=\left(\frac{B}{4 \pi^{2}}+\frac{\chi}{\pi^{2}}\right) \boldsymbol{\omega} \cdot \mathbf{b} .
$$

Alternatively, we can also use $M_{\omega}^{\mathcal{D}}$, which does not involve vacuum shift to give ${ }^{4}$

$$
\Delta J^{0}=-2\left(2 p_{, B^{2}}+M_{\omega, \mu}^{\mathcal{D}}\right) B \omega=\left(\frac{B}{4 \pi^{2}}+\frac{\chi}{\pi^{2}}\right) \boldsymbol{\omega} \cdot \mathbf{b}
$$

We can easily convince ourselves that the structure of the solution $j_{(1) \mathcal{V}}^{\mu} \propto(u+b)^{\mu}$ dictates that

$$
\Delta \mathbf{J}_{A}=\left(\frac{B}{4 \pi^{2}}+\frac{\chi}{\pi^{2}}\right) \boldsymbol{\omega}
$$

The vacuum parts of (4.32) and (4.34) are in agreement with [11]. The medium part for (4.34) is consistent with the standard CVE result.

Let us further work out the frame transformation that connects (4.23) with (4.24). The frame transformation amounts to a redefinition of temperature and chemical potential $T \rightarrow T+\delta T$ and $\mu \rightarrow \mu+\delta \mu$, giving the following matching equations,

$$
\begin{aligned}
f_{\mathcal{N}} & =f_{\mathcal{N}}^{\prime}+\delta T n_{,_{T}}+\delta \mu n_{, \mu}, \\
f_{\mathcal{E}} & =f_{\mathcal{E}}^{\prime}+\delta T \epsilon_{,_{T}}+\delta \mu \epsilon_{, \mu}, \\
f_{\mathcal{P}} & =f_{\mathcal{P}}^{\prime}+\delta T \Pi_{,_{T}}+\delta \mu \Pi_{, \mu}, \\
f_{\mathcal{T}} & ={f^{\prime}}_{\mathcal{T}}+\frac{B^{2}}{3}\left(\delta T \alpha_{B B, T}+\delta \mu \alpha_{B B, \mu}\right) .
\end{aligned}
$$

Now we can plug $M_{\omega}^{\mathcal{V}}$ into (4.35) to get

$$
\begin{aligned}
\delta T n_{,_{T}}+\delta \mu n_{, \mu} & =-\frac{7 B \omega}{12 \pi^{2}}, \\
\delta T p_{,_{T}}+\delta \mu p_{, \mu} & =-\frac{\mu B \omega}{12 \pi^{2}} .
\end{aligned}
$$

The equations can be solved by

$$
\delta T=\frac{3 \omega \mu}{\pi^{2} T}, \quad \delta \mu=-\frac{7 \omega}{6} .
$$

We can now translate the frame transformation back to the following $F_{4}$,

$$
F_{4}=\delta(p \cdot(u+b))\left(\frac{\partial f(p \cdot u)}{\partial T} \delta T+\frac{\partial f(p \cdot u)}{\partial \mu} \delta \mu\right) e^{\frac{p_{T}^{2}}{B}}
$$

\footnotetext{
${ }^{4}$ Though we study vortical and drift perturbations individually at $O(\partial)$, the generation of charge density in vortical solution and generation of heat current in drift solution are connected by Onsager relation [16].
} 
It is instructive to write down the difference of the final solution with $F_{4}$ added and the vacuum solution,

$$
\begin{aligned}
j_{(1) \mathcal{V}}^{\mu}-j_{\text {vac }}^{\mu}= & (u+b)^{\mu}\left[-\frac{\omega}{3}\left(\frac{p_{T}^{2}}{B}+1\right) \delta^{\prime}(p \cdot(u+b)) f(p \cdot u)\right. \\
& +\left(\frac{2 \omega p_{T}^{2}}{3 B}+\frac{\omega}{6}-\frac{3 \omega \mu}{2 \pi^{2} T^{2}}(p \cdot u-\mu)\right) \delta(p \cdot(u+b)) f^{\prime}(p \cdot u) \\
& \left.-\frac{2 \omega p_{T}^{2}}{B^{2}} p \cdot u \delta(p \cdot(u+b)) f(p \cdot u)\right] e^{\frac{p_{T}^{2}}{B}}+\frac{\omega p_{T}^{\mu}}{B} \delta(p \cdot(u+b)) f(p \cdot u) e^{\frac{p_{T}^{2}}{B}}
\end{aligned}
$$

The structure of (4.39) suggests the following interpretation: the first line is modification of dispersion, which does not contribute to charge density upon momenta integration. The terms proportional to $\frac{\omega}{6}$ and $\frac{3 \omega \mu}{2 \pi^{2} T^{2}}(\mu-p \cdot u)$ come from relative shifts of chemical potential $\delta \mu-\delta \mu_{\text {vac }}$ and temperature $\delta T$ respectively. Because the zeroth order charge density $n$ is independent of temperature, contribution to charge density from temperature shift $\delta T n_{, T}$ vanishes. The remaining terms with factors of $p_{T}$ and $p_{T}^{2}$ come from deformation of wave function of the LLL states averaged over the fluid cell, which can be interpreted as excitations of LLL states. Note that higher Landau levels are not excited because they are gapped by $\sqrt{B} \gg \omega$. One may ask whether the vacuum and medium contributions can be traced back to shifts and excitations respectively. In fact, it is not true. The vacuum part is given by the terms $\propto \frac{2 \omega p_{T}^{2}}{3 B}+\frac{\omega}{6}$, which is a mixture of excitations and shifts. The medium part indeed comes from excitations. Finally we remark that we cannot naively take the vacuum limit $T \rightarrow 0$ in our solution because hydrodynamic description breaks down before the limit is reached.

\section{Summary}

We have obtained covariant chiral kinetic theory with Landau level basis. We have used it to study the magnetized plasma subject to transverse electric field. The solution of the Wigner function is the same as the equilibrium one but with a drift velocity just as in system consisting of free fermions. It gives rise to Hall current and heat current.

We have also studied the Wigner function corresponding to a magnetized plasma with a steady vorticity. The resulting solution contains shifts of temperature and chemical potential as well as excitations of the LLL states. It also gives rise to an vector charge density and axial current density. The vacuum parts of both agree with previous studies and medium part of axial current density is consistent with standard CVE result. We find the vacuum contribution comes from the combination of the two effects, while the medium contribution comes from the excitation effect alone.

The current and stress tensor in both cases have been matched to constitutive relations of MHD, allowing us to determine several thermodynamic functions. An apparent discrepancy in the resulting thermodynamic function has been found. The resolution leads to the conclusion that the vacuum state is shifted as the vorticity is turned on adiabatically. The interpretation is in agreement with [11]. 
The expectation that axial current comes solely from LLL states seems to indicate (4.34) is exact to $O(\omega)$. Indeed a same result for charge density is obtained for weak magnetic and vorticity fields based on conventional CKT [65]. The numerical agreement of (4.32) and (4.34) follows from an emergent symmetry in LLL approximation. We expect the vector charge density to receive corrections from high Landau levels in general. It would be interesting to extend the present work to include higher Landau levels.

Last but not least, our study is based on collisionless kinetic theory. There have been indications that vorticity can induce spin rotation of fermions through collision effect [53, 66-69], which could lead to current generation for fermions with anisotropic distribution [70]. It is curious to see whether similar mechanism is manifested with Landau level states. We leave it for future studies.

\section{Acknowledgments}

We thank Han Gao for collaboration at early stage of the work. We are grateful to Jianhua Gao, Koichi Hattori and Yi Yin for useful discussions, also to Koichi Hattori and Yi Yin for helpful comments on an early version of the paper. S.L. thanks Aradhya Shukla for collaborations on related works. S.L. is in part supported by NSFC under Grant Nos 12075328, 11735007 and 11675274.

\section{A Projection}

The decomposition of EOM can be easily done by using the following identity

$$
\sigma^{\mu} \bar{\sigma}^{\nu}=g^{\mu \nu}+\left(g^{\mu \rho} g^{\nu \sigma}-g^{\mu \sigma} g^{\nu \rho}-i \epsilon^{\mu \nu \rho \sigma}\right) n_{\rho}\left(n_{\sigma} n_{\lambda}-g_{\sigma \lambda}\right) \sigma^{\lambda}
$$

Here $n$ can be viewed as a time-like frame vector. $\left(n_{\sigma} n_{\lambda}-g_{\sigma \lambda}\right) \sigma^{\lambda}$ is the spatial components of Pauli matrices orthogonal to $n$. Note that (A.1) splits into an identity part and a Pauli matrix part, both of which contains real and imaginary parts. It is not difficult to see $W(x, p)$ is hermitian from the definition and $\left(\bar{\sigma}^{\mu}\right)^{\dagger}=\bar{\sigma}^{\mu}$. It follows that $j_{\mu}$ is real. Therefore, the splitting gives in total four equations

$$
\begin{aligned}
\Pi_{\mu} j^{\mu} & =0 \\
\Delta_{\mu} j^{\mu} & =0 \\
\Pi^{\mu} j^{\nu}-\Pi^{\nu} j^{\mu} & =-\frac{1}{2} \epsilon^{\mu \nu \rho \sigma} \Delta_{\rho} j_{\sigma}, \\
\frac{1}{2}\left(\Delta^{\mu} j^{\nu}-\Delta^{\nu} j^{\mu}\right) & =\epsilon^{\mu \nu \rho \sigma} \Pi_{\rho} j_{\sigma}, .
\end{aligned}
$$

In fact (A.5) is equivalent to (A.4).

The case of left-handed fermions is similar. The only difference is that we replace $\sigma$ in the covariant derivative (2.2) by $\bar{\sigma}$ and $\bar{\sigma}$ in the decomposition (2.10) by $\sigma$, which simply flips the sign of r.h.s. of (A.4) and (A.5). 


\section{B Momenta calculus}

We show the momenta differentiation and integration in detail with $q^{\mu}=\Lambda_{\nu}^{\mu} p^{\nu}$ as independent variables and

$$
\Lambda_{\nu}^{\mu}=\left(u^{\nu},-w_{i}^{\nu},-b^{\nu}\right)^{T}, \quad \Lambda_{\nu}^{-1 \mu}=\left(u^{\mu}, w_{i}^{\mu}, b^{\mu}\right),
$$

being Lorentz transformations between $p^{\mu}$ and $q^{\mu}$. We note $\Lambda_{\mu}^{0}=\Lambda_{0}^{-1 \mu}=u^{\mu}, \Lambda_{\mu}^{i}=\Lambda_{i}^{-1 \mu}=$ $w_{i}^{\mu}$ with transverse index $i=1,2$ and $\Lambda_{\mu}^{3}=\Lambda_{3}^{-1 \mu}=b^{\mu}$ are basis column vectors which are orthogonal to one another and normalized as $u^{2}=1, w_{i}^{2}=b^{2}=-1$. The metric in most minus signature can be written as $g^{\mu \nu}=u^{\mu} u^{\nu}-w_{i}^{\mu} w_{i}^{\nu}-b^{\mu} b^{\nu}$ with summation over $i=1,2$. Then $p^{2}=(p \cdot u)^{2}-\left(p \cdot w_{i}\right)\left(p \cdot w_{i}\right)-(p \cdot b)^{2} \equiv q_{0}^{2}-q_{T}^{2}-q_{3}^{2}=q^{2}$ with $q_{0} \equiv p \cdot u, q_{i} \equiv-p \cdot w_{i}, q_{3} \equiv-p \cdot b$, which gives $p_{T}^{\mu}=q_{i} w_{i}^{\mu}$ and $p_{T}^{2}=-q_{T}^{2}$.

Note there are gradients in $q_{0}=p \cdot u$ and $q_{T}^{2}=-p_{T}^{2}$ since they depend on $u^{\mu}$. Explicitly, $\partial_{\mu}(p \cdot u)=p^{\lambda} \partial_{\mu} u_{\lambda}$ and $\partial_{\mu} p_{T}^{2}=\partial_{\mu}\left(p^{2}-(p \cdot u)^{2}+(p \cdot b)^{2}\right)=-2 p \cdot u p^{\lambda} \partial_{\mu} u_{\lambda}$. Moreover, $p_{T}^{\mu}, E^{\mu}$ and $u_{(1)}^{\mu}$ lie in the transverse plane spanned by $w_{i}^{\mu}$ and therefore give vanishing dot products with $u^{\mu}$ and $b^{\mu}$. We repeatedly use these properties in the text and the following calculations.

The momenta differentiation can be interpreted as

$$
\frac{\partial}{\partial p^{\mu}}=\frac{\partial}{\partial(p \cdot u)} u_{\mu}+\frac{\partial}{\partial(p \cdot b)} b_{\mu}+\frac{\partial}{\partial\left(p \cdot w_{i}\right)} w_{\mu}^{i}
$$

Multiplying it by $u^{\mu}$ and $b^{\mu}$, one gets $u^{\mu} \frac{\partial}{\partial p^{\mu}}=\frac{\partial}{\partial(p \cdot u)}$ and $b^{\mu} \frac{\partial}{\partial p^{\mu}}=-\frac{\partial}{\partial(p \cdot b)}$. We can then write the operator $\delta \Pi_{\mu} \equiv \Pi_{\mu}-p_{\mu}=-\frac{1}{12}\left(\partial_{\lambda}^{p} \partial_{x}^{\lambda}\right) \frac{\partial}{\partial p_{\nu}} F_{\mu \nu}$ explicitly as follows. Using $\partial^{\lambda} u^{\rho}=-\omega \epsilon^{\lambda \rho \alpha \beta} b_{\alpha} u_{\beta}$, we get

$$
\begin{aligned}
\delta \Pi_{\mu} & =\frac{B \omega}{12} \frac{\partial}{\partial p_{\lambda}} \frac{\partial}{\partial p_{\nu}}\left(b_{\lambda} b_{\mu} u_{\nu}-b_{\lambda} b_{\nu} u_{\mu}+g_{\lambda \mu} u_{\nu}-g_{\lambda \nu} u_{\mu}\right) \\
& =\frac{B \omega}{12}\left[\frac{\partial}{\partial(p \cdot u)}\left(\frac{\partial}{\partial p^{\mu}}-\frac{\partial}{\partial(p \cdot b)} b_{\mu}\right)-\frac{\partial}{\partial p_{\lambda}} \frac{\partial}{\partial p_{\nu}}\left(g_{\lambda \nu}+b_{\lambda} b_{\nu}\right) u_{\mu}\right] .
\end{aligned}
$$

Applying the chain rule (B.2) in the first part, we obtain

$$
\begin{aligned}
\delta \Pi_{\mu} & =\frac{B \omega}{12}\left[\frac{\partial}{\partial(p \cdot u)}\left(\frac{\partial}{\partial(p \cdot u)} u_{\mu}+\frac{\partial}{\partial\left(p \cdot w_{i}\right)} w_{\mu}^{i}\right)-\frac{\partial}{\partial p_{\lambda}} \frac{\partial}{\partial p_{\nu}}\left(g_{\lambda \nu}+b_{\lambda} b_{\nu}\right) u_{\mu}\right] \\
& =\frac{B \omega}{12}\left[\frac{\partial}{\partial(p \cdot u)} \frac{\partial}{\partial\left(p \cdot w_{i}\right)} w_{\mu}^{i}-\frac{\partial}{\partial p_{\lambda}} \frac{\partial}{\partial p_{\nu}}\left(g_{\lambda \nu}+b_{\lambda} b_{\nu}-u_{\lambda} u_{\nu}\right) u_{\mu}\right] \\
& =\frac{B \omega}{12}\left(\frac{2 p_{\mu}^{T}}{B} \frac{\partial}{\partial(p \cdot u)}+u_{\mu} P_{\lambda \nu} \frac{\partial}{\partial p_{\lambda}} \frac{\partial}{\partial p_{\nu}}\right)
\end{aligned}
$$

where, in the last equality, we have identified the operator $\frac{\partial}{\partial\left(p \cdot w_{i}\right)} w_{\mu}^{i}=\frac{2 p_{\mu}^{T}}{B}$ upon acting on functions depending on $p_{T}^{\mu}$ through $e^{\frac{p_{T}^{2}}{B}}$ only. Also, we have let $\frac{\partial}{\partial(p \cdot u)}$ pass over $\frac{2 p_{\mu}^{T}}{B}$ since $p_{\mu}^{T}$ is independent of $p \cdot u$. 
When solving the kinetic equations, we have repeatedly used the vanishing integration by parts

$$
\begin{array}{r}
p \cdot(u+b) \delta^{\prime}(p \cdot(u+b))+\delta(p \cdot(u+b))=0, \\
\text { or } \quad\left(q_{0}-q_{3}\right) \delta^{\prime}\left(q_{0}-q_{3}\right)+\delta\left(q_{0}-q_{3}\right)=0,
\end{array}
$$

To include contribution from left-handed fermions, we generalize (2.13) and (2.14) as

$$
\begin{aligned}
j_{s} & =\delta\left(q_{0}-s q_{3}\right) f_{s}\left(q_{0}\right) e^{\frac{-q_{T}^{2}}{B}}, \\
f_{s}\left(q_{0}\right) & =\frac{2}{(2 \pi)^{3}} \sum_{r= \pm} \frac{r \theta\left(r q_{0}\right)}{e^{r\left(q_{0}-\mu_{s}\right) / T}+1}
\end{aligned}
$$

where helicity $s= \pm$ with $\mu_{+}=\mu_{R}$ and $\mu_{-}=\mu_{L}$ respectively. With shorthand notations $\int \equiv \int d q_{0} d q_{3}, \delta_{s} \equiv \delta\left(q_{0}-s q_{3}\right)$ and $f_{s} \equiv f_{s}\left(q_{0}\right)$, the following integrals are useful to perform momenta integration

$$
\begin{aligned}
\int\left(\delta_{s} f_{s}\right)^{\prime} & =-1, \quad \int \delta_{s} f_{s}^{\prime}=-1, \rightarrow \int \delta_{s}^{\prime} f_{s}=0 \\
\int\left(q_{0} \delta_{s} f_{s}\right)^{\prime} & =0, \quad \int \delta_{s} f_{s}=\mu_{s}, \rightarrow \int q_{0}\left(\delta_{s} f_{s}\right)^{\prime}=-\mu_{s} \\
\int q_{0} \delta_{s} f_{s}^{\prime} & =-\mu_{s}, \rightarrow \int q_{0} \delta_{s}^{\prime} f_{s}=0, \quad \int s q_{3} \delta_{s} f_{s}^{\prime}=-\mu_{s}, \quad \rightarrow \int s q_{3} \delta_{s}^{\prime} f_{s}=\mu_{s}, \\
\int q_{0} \delta_{s} f_{s} & =\int s q_{3} \delta_{s} f_{s}=\frac{\mu_{s}^{2}}{2}+\frac{\pi^{2} T^{2}}{6}, \\
\int q_{0}^{2} \delta_{s} f_{s} & =\int s q_{3} q_{0} \delta_{s} f_{s}=\frac{\mu_{s}}{3}\left(\mu_{s}^{2}+\pi^{2} T^{2}\right) \\
\int d q_{1} d q_{2} e^{\frac{-q_{T}^{2}}{B}} & =\int d q_{1} d q_{2} \frac{q_{T}^{2}}{B} e^{\frac{-q_{T}^{2}}{B}}=2 \int d q_{1} d q_{2} \frac{q_{i}^{2}}{B} e^{\frac{-q_{T}^{2}}{B}}=\pi B .
\end{aligned}
$$

Then, for the $O(1)$ solution, we have

$$
\begin{aligned}
u_{\mu} J_{(0)}^{\mu} & =\int d^{4} p u_{\mu} j_{(0)}^{\mu}=\int d^{4} q \sum_{s= \pm} j_{s}=\frac{\mu B}{2 \pi^{2}} \\
P^{\mu \nu} J_{\nu}^{(0)} & =\int d^{4} p P^{\mu \nu} j_{\nu}^{(0)}=0 \\
b^{\mu} b^{\nu} J_{\nu}^{(0)} & =\int d^{4} p b^{\mu} b^{\nu} j_{\nu}^{(0)}=-\int d^{4} q \sum_{s= \pm} s b^{\mu} j_{s}=-\frac{\mu_{5} B}{2 \pi^{2}} b^{\mu} \\
u_{\mu} u_{\nu} T_{(0)}^{\mu \nu} & =\int d^{4} p u_{\mu} u_{\nu} \frac{1}{2} p^{\{\mu} j_{(0)}^{\nu\}}=\int d^{4} q q_{0} \sum_{s= \pm} j_{s}=\frac{\chi_{V} B}{2 \pi^{2}} \\
P_{\mu \nu} T_{(0)}^{\mu \nu} & =\int d^{4} p P_{\mu \nu} \frac{1}{2} p^{\{\mu} j_{(0)}^{\nu\}}=0, \\
b_{\mu} b_{\nu} T_{(0)}^{\mu \nu} & =\int d^{4} p b_{\mu} b_{\nu} \frac{1}{2} p^{\{\mu} j_{(0)}^{\nu\}}=\int d^{4} q \sum_{s= \pm} s q_{3} j_{s}=\frac{\chi_{V} B}{2 \pi^{2}}
\end{aligned}
$$




$$
\begin{aligned}
\Delta^{\mu \alpha} \Delta^{\nu \beta} T_{\alpha \beta}^{(0)} & =\int d^{4} p b^{\mu} b^{\alpha} b^{\nu} b^{\beta} \frac{1}{2} p_{\{\alpha} j_{\beta\}}^{(0)}=\frac{\omega}{B} \int d^{4} q \sum_{s= \pm} b^{\mu} b^{\nu} s q_{3} j_{s}=\frac{\omega}{2 \pi^{2}} \chi_{V} b^{\mu} b^{\nu}, \\
P^{\mu \alpha} u^{\beta} T_{\alpha \beta}^{(0)} & =\int d^{4} p P^{\mu \alpha} u^{\beta} p_{\{\alpha} j_{\beta\}}^{(0)}=0, \\
b^{\mu} b^{\alpha} u^{\beta} T_{\alpha \beta}^{(0)} & =\int d^{4} p b^{\mu} b^{\alpha} u^{\beta} \frac{1}{2} p_{\{\alpha} j_{\beta\}}^{(0)}=-b^{\mu} \int d^{4} q \sum_{s= \pm}\left(q_{3}+s q_{0}\right) j_{s}=\frac{-\chi_{A}}{2 \pi^{2}} b^{\mu},
\end{aligned}
$$

which give

$$
\begin{aligned}
& \mathcal{N}_{(0)}=\frac{\mu B}{2 \pi^{2}}, \quad \mathcal{J}_{(0)}^{\mu}=-\Delta^{\mu \nu} J_{\nu}^{(0)}=\frac{\mu_{5} B}{2 \pi^{2}} b^{\mu}, \\
& \mathcal{E}_{(0)}=\frac{\chi_{V} B}{2 \pi^{2}}, \quad \mathcal{P}_{(0)}=\frac{1}{3} \Delta_{\mu \nu} T_{(0)}^{\mu \nu}=\frac{\chi_{V} B}{6 \pi^{2}}, \quad \mathcal{Q}_{(0)}^{\mu}=\Delta^{\mu \alpha} u^{\beta} T_{\alpha \beta}^{(0)}=-\frac{\chi_{A}}{2 \pi^{2}} b^{\mu}, \\
& \mathcal{T}_{(0)}^{\mu \nu}=\frac{1}{2}\left(\Delta^{\mu \alpha} \Delta^{\nu \beta}+\Delta^{\nu \alpha} \Delta^{\mu \beta}-\frac{2}{3} \Delta^{\mu \nu} \Delta^{\alpha \beta}\right) T_{\alpha \beta}^{(0)}=\frac{\chi_{V} B}{6 \pi^{2}}\left(2 b^{\mu} b^{\nu}-P^{\mu \nu}\right) .
\end{aligned}
$$

The following are $O(\partial)$ solutions. Firstly, for drift solution, the nontrivial components are

$$
\begin{aligned}
\mathcal{J}_{(1) \mathcal{D}}^{\mu} & =-\Delta^{\mu \nu} J_{\nu}^{(1) \mathcal{D}}=-\int d^{4} p P^{\mu \nu} j_{\nu}^{(1) \mathcal{D}}=-\int d^{4} p P^{\mu \nu} \sum_{s= \pm} u_{\nu}^{(1)} j_{s} \\
& =\int d^{4} q u_{(1)}^{\mu} \sum_{s= \pm} j_{s}=\frac{\mu}{4 \pi^{2}} \epsilon^{\mu \nu \rho \sigma} f_{\nu \rho} b_{\sigma}=-\frac{\mu}{2 \pi^{2}} \epsilon^{\mu \nu \rho \sigma} u_{\nu} E_{\rho} b_{\sigma} \\
\mathcal{Q}_{(1) \mathcal{D}}^{\mu} & =-\Delta^{\mu \alpha} u^{\beta} T_{\alpha \beta}^{(1) \mathcal{D}}=-\int d^{4} p P^{\mu \alpha} u^{\beta} \frac{1}{2} p_{\{\alpha} j_{\beta\}}^{(1) \mathcal{D}} \\
& =\frac{1}{2} \int d^{4} p \sum_{s= \pm}\left[p \cdot u_{(1)} p_{T}^{\mu}\left(\frac{\partial}{\partial(p \cdot u)}-\frac{2 p \cdot u}{B}\right) j_{s}+p \cdot u u_{(1)}^{\mu} j_{s}\right] \\
& =\frac{1}{2} \int d^{4} q u_{(1)}^{\mu} \sum_{s= \pm}\left[\frac{q_{T}^{2}}{2}\left(\frac{2 q_{0}}{B}-\frac{\partial}{\partial q_{0}}\right) j_{s}+q_{0} j_{s}\right] \\
& =\frac{1}{2 \pi^{2}}\left(\frac{B}{4}+\chi_{V}\right) u_{(1)}^{\mu} \\
& =-\frac{1}{2 \pi^{2}}\left(\frac{B}{4}+\chi_{V}\right) \epsilon^{\mu \nu \rho \sigma} u_{\nu} E_{\rho} b_{\sigma} .
\end{aligned}
$$

All the other components from drift solution are vanishing upon integration over $q_{i}$ odd functions. For vortical solution, we have

$$
\begin{aligned}
u_{\mu} J_{(1) \mathcal{C}}^{\mu} & =\int d^{4} p u_{\mu} j_{(1) \mathcal{C}}^{\mu}=\frac{\omega}{B} \int d^{4} q \sum_{s= \pm} q_{T}^{2}\left(\frac{2 q_{0}}{B} j_{s}-j_{s}^{\prime}+\frac{1}{3} \delta_{s} f_{s}^{\prime} e^{\frac{-q_{T}^{2}}{B}}\right)=\frac{\omega}{2 \pi^{2}}\left(2 \chi_{V}+\frac{2}{3} B\right), \\
P^{\mu \nu} J_{\nu}^{(1) \mathcal{C}} & =\int d^{4} p P^{\mu \nu} j_{\nu}^{(1) \mathcal{C}}=0 \\
b^{\mu} b^{\nu} J_{\nu}^{(1) \mathcal{C}} & =\int d^{4} p b^{\mu} b^{\nu} j_{\nu}^{(1) \mathcal{C}}=-\frac{\omega}{B} b^{\mu} \int d^{4} q \sum_{s= \pm} s q_{T}^{2}\left(\frac{2 q_{0}}{B} j_{s}-j_{s}^{\prime}+\frac{1}{3} \delta_{s} f_{s}^{\prime} e^{\frac{-q_{T}^{2}}{B}}\right) \\
& =-\frac{\omega}{2 \pi^{2}} 2 \chi_{A} b^{\mu} \\
u_{\mu} u_{\nu} T_{(1) \mathcal{C}}^{\mu \nu} & =\int d^{4} p u_{\mu} u_{\nu} \frac{1}{2} p^{\{\mu} j_{(1) \mathcal{C}}^{\nu\}}=\frac{\omega}{B} \int d^{4} q \sum_{s= \pm} q_{0} q_{T}^{2}\left(\frac{2 q_{0}}{B} j_{s}-j_{s}^{\prime}+\frac{1}{3} \delta_{s} f_{s}^{\prime} e^{\frac{-q_{T}^{2}}{B}}\right) \\
& =\frac{\omega}{2 \pi^{2}}\left(2 \xi_{V}+\frac{2}{3} \mu B\right),
\end{aligned}
$$




$$
\begin{aligned}
P_{\mu \nu} T_{(1) \mathcal{C}}^{\mu \nu} & =\int d^{4} p P_{\mu \nu} \frac{1}{2} p^{\{\mu} j_{(1) \mathcal{C}}^{\nu\}}=\frac{\omega}{B} \int d^{4} q \sum_{s= \pm} q_{T}^{2} j_{s}=\frac{\omega}{2 \pi^{2}} \mu B \\
b_{\mu} b_{\nu} T_{(1) \mathcal{C}}^{\mu \nu} & =\int d^{4} p b_{\mu} b_{\nu} \frac{1}{2} p^{\{\mu} j_{(1) \mathcal{C}}^{\nu\}}=\frac{\omega}{B} \int d^{4} q \sum_{s= \pm} s q_{3} q_{T}^{2}\left(\frac{2 q_{0}}{B} j_{s}-j_{s}^{\prime}+\frac{1}{3} \delta_{s} f_{s}^{\prime} e^{\frac{-q_{T}^{2}}{B}}\right) \\
& =\frac{\omega}{2 \pi^{2}}\left(2 \xi_{V}-\frac{1}{3} \mu B\right), \\
\Delta^{\mu \alpha} \Delta^{\nu \beta} T_{\alpha \beta}^{(1) \mathcal{C}}= & \int d^{4} p\left(P^{\mu \alpha} P^{\nu \beta}+b^{\mu} b^{\alpha} b^{\nu} b^{\beta}\right) \frac{1}{2} p_{\{\alpha} j_{\beta\}}^{(1) \mathcal{C}} \\
& =\frac{\omega}{B} \int d^{4} p \sum_{s= \pm}\left[p_{T}^{\mu} p_{T}^{\nu} j_{s}+b^{\mu} b^{\nu}(-s p \cdot b) p_{T}^{2}\left(\frac{\partial j_{s}}{\partial(p \cdot u)}-\frac{1}{3} \delta_{s} f_{s} e^{\frac{p_{T}^{2}}{B}}-\frac{2 p \cdot u}{B} j_{s}\right)\right] \\
& =\frac{\omega}{B} \int d^{4} q \sum_{s= \pm}\left[\frac{1}{2} P^{\mu \nu} q_{T}^{2} j_{s}+b^{\mu} b^{\nu} s q_{3} q_{T}^{2}\left(\frac{2 q_{0}}{B} j_{s}-j_{s}^{\prime}+\frac{1}{3} \delta_{s} f_{s}^{\prime} e^{\frac{-q_{T}^{2}}{B}}\right)\right] \\
& =\frac{1}{2} P^{\mu \nu} \frac{\omega}{2 \pi^{2}} \mu B+b^{\mu} b^{\nu} \frac{\omega}{2 \pi^{2}}\left(2 \xi_{V}-\frac{1}{3} \mu B\right), \\
P^{\mu \alpha} u^{\beta} T_{\alpha \beta}^{(1) \mathcal{C}}= & \int d^{4} p P^{\mu \alpha} u^{\beta} \frac{1}{2} p_{\{\alpha} j_{\beta\}}^{(1) \mathcal{C}}=0, \\
b^{\mu} b^{\alpha} u^{\beta} T_{\alpha \beta}^{(1) \mathcal{C}}= & \int d^{4} p b^{\mu} b^{\alpha} u^{\beta} \frac{1}{2} p_{\{\alpha} j_{\beta\}}^{(1) \mathcal{C}}=-\frac{b^{\mu} \omega}{2 B} \int d^{4} q \sum_{s= \pm}\left(q_{3}+s q_{0}\right) \\
& \times q_{T}^{2}\left(\frac{2 q_{0}}{B} j_{s}-j_{s}^{\prime}+\frac{1}{3} \delta_{s} f_{s}^{\prime} e^{\frac{-q_{T}^{2}}{B}}\right)=-\frac{\omega}{2 \pi^{2}}\left(2 \xi_{A}+\frac{1}{6} \mu_{5} B\right) b^{\mu} .
\end{aligned}
$$

The $j_{(1) \mathcal{A}}^{\mu}$ part does not contribute to the final vortical result since we have

$$
\delta \Pi_{u} e^{\frac{p_{T}^{2}}{B}} \sim P_{\lambda \nu} \frac{\partial}{\partial p_{\lambda}} \frac{\partial}{\partial p_{\nu}} e^{\frac{p_{T}^{2}}{B}}=\frac{\partial}{\partial q_{i}} \frac{\partial}{\partial q_{i}} e^{\frac{-q_{T}^{2}}{B}}=\frac{4}{B}\left(\frac{q_{T}^{2}}{B}-1\right) e^{\frac{-q_{T}^{2}}{B}},
$$

which gives vanishing integral by noting $\int d q_{1} d q_{2} e^{\frac{-q_{T}^{2}}{B}}=\int d q_{1} d q_{2} \frac{q_{T}^{2}}{B} e^{\frac{-q_{T}^{2}}{B}}$. Thus

$$
\begin{aligned}
\mathcal{N}_{(1) \mathcal{V}} & =\frac{\omega}{2 \pi^{2}}\left(2 \chi_{V}+\frac{2}{3} B\right), \quad \mathcal{J}_{(1) \mathcal{V}}^{\mu}=-\Delta^{\mu \nu} J_{\nu}^{(1) \mathcal{V}}=\frac{\omega}{2 \pi^{2}} 2 \chi_{A} b^{\mu} \\
\mathcal{E}_{(1) \mathcal{V}} & =\frac{\omega}{2 \pi^{2}}\left(2 \xi_{V}+\frac{2}{3} \mu B\right), \quad \mathcal{P}_{(1) \mathcal{V}}=\Delta_{\mu \nu} T_{(1) \mathcal{V}}^{\mu \nu}=\frac{\omega}{6 \pi^{2}}\left(2 \xi_{V}+\frac{2}{3} \mu B\right) \\
\mathcal{Q}_{(1) \mathcal{V}}^{\mu} & =-\Delta^{\mu \alpha} u^{\beta} T_{\alpha \beta}^{(1) \mathcal{V}}=\frac{\omega}{2 \pi^{2}}\left(2 \xi_{A}+\frac{1}{6} \mu_{5} B\right) b^{\mu} \\
\mathcal{T}_{(1) \mathcal{V}}^{\mu \nu} & =\frac{1}{2}\left(\Delta^{\mu \alpha} \Delta^{\nu \beta}+\Delta^{\nu \alpha} \Delta^{\mu \beta}-\frac{2}{3} \Delta^{\mu \nu} \Delta^{\alpha \beta}\right) T_{\alpha \beta}^{(1) \mathcal{V}} \\
& =\frac{\omega}{6 \pi^{2}}\left(2 \xi_{V}-\frac{5}{6} \mu B\right)\left(2 b^{\mu} b^{\nu}-P^{\mu \nu}\right) .
\end{aligned}
$$




\section{Useful formulas}

We have repeatedly used the contraction formulas of two anti-symmetric tensors,

$$
\begin{gathered}
\epsilon^{\mu \nu \rho \sigma} \epsilon_{\mu \nu \alpha \beta}=-2\left|\begin{array}{cc}
\delta_{\alpha}^{\rho} & \delta_{\beta}^{\rho} \\
\delta_{\alpha}^{\sigma} & \delta_{\beta}^{\sigma}
\end{array}\right|, \\
\epsilon^{\mu \nu \rho \sigma} \epsilon_{\mu \lambda \alpha \beta}=-\left|\begin{array}{lll}
\delta_{\lambda}^{\nu} & \delta_{\alpha}^{\nu} & \delta_{\beta}^{\nu} \\
\delta_{\lambda}^{\rho} & \delta_{\alpha}^{\rho} & \delta_{\beta}^{\rho} \\
\delta_{\lambda}^{\sigma} & \delta_{\alpha}^{\sigma} & \delta_{\beta}^{\sigma}
\end{array}\right|
\end{gathered}
$$

By repeated use of the five-index cyclic identity,

$$
\epsilon^{\mu \nu \rho \sigma} p^{\lambda}+\epsilon^{\nu \rho \sigma \lambda} p^{\mu}+\epsilon^{\rho \sigma \lambda \mu} p^{\nu}+\epsilon^{\sigma \lambda \mu \nu} p^{\rho}+\epsilon^{\lambda \mu \nu \rho} p^{\sigma}=0
$$

we can prove the identities in (3.10). For the first one,

$$
\epsilon^{\mu \nu \rho \sigma} f_{\rho \lambda}(u+b)_{\sigma} u^{\lambda}=-\left(\epsilon^{\nu \rho \sigma \lambda} u^{\mu}+\epsilon^{\rho \sigma \lambda \mu} u^{\nu}+\epsilon^{\sigma \lambda \mu \nu} u^{\rho}+\epsilon^{\lambda \mu \nu \rho} u^{\sigma}\right) f_{\rho \lambda}(u+b)_{\sigma} .
$$

Upon moving the third term $\epsilon^{\sigma \lambda \mu \nu} u^{\rho}$ of the r.h.s. to the l.h.s., one finds the l.h.s. doubles. At the same time, the $u_{\sigma}$ parts in the first and second terms vanish by noting $f_{\rho \lambda}=$ $E_{\rho} u_{\lambda}-E_{\lambda} u_{\rho}$ and the anti-symmetry of $\epsilon^{\nu \rho \sigma \lambda}$ and $\epsilon^{\rho \sigma \lambda \mu}$. Rearranging the indices, one gets

$$
2 \epsilon^{\mu \nu \rho \sigma} f_{\rho \lambda}(u+b)_{\sigma} u^{\lambda}=u^{[\mu} \epsilon^{\nu] \rho \lambda \sigma} f_{\rho \lambda} b_{\sigma}+\epsilon^{\mu \nu \rho \lambda} f_{\rho \lambda} .
$$

The $\epsilon^{\mu \nu \rho \lambda} f_{\rho \lambda}$ term can be written into $b^{[\mu} \epsilon^{\nu] \rho \sigma \lambda} f_{\rho \lambda} b_{\sigma}$ by starting from

$$
b^{\mu} \epsilon^{\nu \rho \lambda \sigma} f_{\rho \lambda} b_{\sigma}=-\left(b^{\nu} \epsilon^{\rho \lambda \sigma \mu}+b^{\rho} \epsilon^{\lambda \sigma \mu \nu}+b^{\lambda} \epsilon^{\sigma \mu \nu \rho}+b^{\sigma} \epsilon^{\mu \nu \rho \lambda}\right) f_{\rho \lambda} b_{\sigma},
$$

where we can move the first term $b^{\nu} \epsilon^{\rho \lambda \sigma \mu}$ of the r.h.s. to the l.h.s. to produce $b^{[\mu} \epsilon^{\nu] \rho \lambda \sigma} f_{\rho \lambda} b_{\sigma}$ in the l.h.s. Then by noting $b^{\rho} f_{\rho \lambda}=b^{\lambda} f_{\rho \lambda}=0$ in the second and third terms, we do get $\epsilon^{\mu \nu \rho \lambda} f_{\rho \lambda}=b^{[\mu} \epsilon^{\nu] \rho \lambda \sigma} f_{\rho \lambda} b_{\sigma}$ which gives

$$
2 \epsilon^{\mu \nu \rho \sigma} f_{\rho \lambda}(u+b)_{\sigma} u^{\lambda}=(u+b)^{[\mu} \epsilon^{\nu] \rho \lambda \sigma} f_{\rho \lambda} b_{\sigma}=2 B(u+b)^{[\mu} u_{(1)}^{\nu]} .
$$

Similarly, for the second identity in (3.10), one has

$$
\begin{aligned}
\epsilon^{\mu \nu \rho \sigma} f_{\rho \lambda}(u+b)_{\sigma} p_{T}^{\lambda} & =-\left(\epsilon^{\nu \rho \sigma \lambda} p_{T}^{\mu}+\epsilon^{\rho \sigma \lambda \mu} p_{T}^{\nu}+\epsilon^{\sigma \lambda \mu \nu} p_{T}^{\rho}+\epsilon^{\lambda \mu \nu \rho} p_{T}^{\sigma}\right) f_{\rho \lambda}(u+b)_{\sigma} \\
\rightarrow \quad 2 \epsilon^{\mu \nu \rho \sigma} f_{\rho \lambda}(u+b)_{\sigma} p_{T}^{\lambda} & =p_{T}^{[\mu} \epsilon^{\nu] \rho \lambda \sigma} f_{\rho \lambda} b_{\sigma}=2 p_{T}^{[\mu} u_{(1)}^{\nu]} .
\end{aligned}
$$

Open Access. This article is distributed under the terms of the Creative Commons Attribution License (CC-BY 4.0), which permits any use, distribution and reproduction in any medium, provided the original author(s) and source are credited. 


\section{References}

[1] A. Vilenkin, Equilibrium parity violating current in a magnetic field, Phys. Rev. D 22 (1980) 3080 [INSPIRE].

[2] D. Kharzeev, Parity violation in hot QCD: why it can happen, and how to look for it, Phys. Lett. B 633 (2006) 260 [hep-ph/0406125] [INSPIRE].

[3] D. Kharzeev and A. Zhitnitsky, Charge separation induced by P-odd bubbles in QCD matter, Nucl. Phys. A 797 (2007) 67 [arXiv:0706.1026] [InSPIRE].

[4] K. Fukushima, D.E. Kharzeev and H.J. Warringa, The chiral magnetic effect, Phys. Rev. D 78 (2008) 074033 [arXiv:0808.3382] [INSPIRE].

[5] D.T. Son and P. Surowka, Hydrodynamics with triangle anomalies, Phys. Rev. Lett. 103 (2009) 191601 [arXiv:0906 .5044] [INSPIRE].

[6] Y. Neiman and Y. Oz, Relativistic hydrodynamics with general anomalous charges, JHEP 03 (2011) 023 [arXiv: 1011.5107] [INSPIRE].

[7] A. Vilenkin, Quantum field theory at finite temperature in a rotating system, Phys. Rev. D 21 (1980) 2260 [INSPIRE].

[8] J. Erdmenger, M. Haack, M. Kaminski and A. Yarom, Fluid dynamics of R-charged black holes, JHEP 01 (2009) 055 [arXiv: 0809.2488] [INSPIRE].

[9] N. Banerjee, J. Bhattacharya, S. Bhattacharyya, S. Dutta, R. Loganayagam and P. Surowka, Hydrodynamics from charged black branes, JHEP 01 (2011) 094 [arXiv:0809.2596] [INSPIRE].

[10] K. Landsteiner, E. Megias and F. Pena-Benitez, Gravitational anomaly and transport, Phys. Rev. Lett. 107 (2011) 021601 [arXiv:1103.5006] [InSPIRE].

[11] K. Hattori and Y. Yin, Charge redistribution from anomalous magnetovorticity coupling, Phys. Rev. Lett. 117 (2016) 152002 [arXiv:1607.01513] [INSPIRE].

[12] Y. Liu and I. Zahed, Pion condensation by rotation in a magnetic field, Phys. Rev. Lett. 120 (2018) 032001 [arXiv:1711.08354] [INSPIRE].

[13] H.-L. Chen, K. Fukushima, X.-G. Huang and K. Mameda, Analogy between rotation and density for Dirac fermions in a magnetic field, Phys. Rev. D 93 (2016) 104052 [arXiv: 1512.08974] [INSPIRE].

[14] G. Cao and L. He, Rotation induced charged pion condensation in a strong magnetic field: a Nambu-Jona-Lasino model study, Phys. Rev. D 100 (2019) 094015 [arXiv:1910.02728] [INSPIRE].

[15] H.-L. Chen, X.-G. Huang and K. Mameda, Do charged pions condense in a magnetic field with rotation?, arXiv: 1910.02700 [INSPIRE].

[16] Y. Bu and S. Lin, Magneto-vortical effect in strongly coupled plasma, Eur. Phys. J. C 80 (2020) 401 [arXiv: 1912.11277] [InSPIRE].

[17] K. Fukushima, T. Shimazaki and L. Wang, Mode decomposed chiral magnetic effect and rotating fermions, Phys. Rev. D 102 (2020) 014045 [arXiv:2004.05852] [INSPIRE].

[18] P. Kovtun, Thermodynamics of polarized relativistic matter, JHEP 07 (2016) 028 [arXiv: 1606.01226] [INSPIRE]. 
[19] J. Hernandez and P. Kovtun, Relativistic magnetohydrodynamics, JHEP 05 (2017) 001 [arXiv: 1703.08757] [INSPIRE].

[20] S. Grozdanov, D.M. Hofman and N. Iqbal, Generalized global symmetries and dissipative magnetohydrodynamics, Phys. Rev. D 95 (2017) 096003 [arXiv:1610.07392] [InSPIRE].

[21] M. Hongo and K. Hattori, Revisiting relativistic magnetohydrodynamics from quantum electrodynamics, JHEP 02 (2021) 011 [arXiv:2005.10239] [INSPIRE].

[22] K. Hattori, Y. Hirono, H.-U. Yee and Y. Yin, MagnetoHydrodynamics with chiral anomaly: phases of collective excitations and instabilities, Phys. Rev. D 100 (2019) 065023 [arXiv: 1711.08450] [INSPIRE].

[23] X.-G. Huang, A. Sedrakian and D.H. Rischke, Kubo formulae for relativistic fluids in strong magnetic fields, Annals Phys. 326 (2011) 3075 [arXiv:1108.0602] [InSPIRE].

[24] S.I. Finazzo, R. Critelli, R. Rougemont and J. Noronha, Momentum transport in strongly coupled anisotropic plasmas in the presence of strong magnetic fields, Phys. Rev. D 94 (2016) 054020 [Erratum ibid. 96 (2017) 019903] [arXiv: 1605.06061] [INSPIRE].

[25] M. Buzzegoli, Thermodynamic equilibrium of massless fermions with vorticity, chirality and electromagnetic field, arXiv:2011.09974 [INSPIRE].

[26] S. Lin and L. Yang, Chiral kinetic theory from Landau level basis, Phys. Rev. D 101 (2020) 034006 [arXiv: 1909.11514] [INSPIRE].

[27] H. Gao, Z. Mo and S. Lin, Photon self-energy in a magnetized chiral plasma from kinetic theory, Phys. Rev. D 102 (2020) 014011 [arXiv:2002.07959] [InSPIRE].

[28] K. Hattori, S. Li, D. Satow and H.-U. Yee, Longitudinal conductivity in strong magnetic field in perturbative QCD: complete leading order, Phys. Rev. D 95 (2017) 076008 [arXiv: 1610.06839] [INSPIRE].

[29] X.-L. Sheng, D.H. Rischke, D. Vasak and Q. Wang, Wigner functions for fermions in strong magnetic fields, Eur. Phys. J. A $\mathbf{5 4}$ (2018) 21 [arXiv:1707.01388] [INSPIRE].

[30] K. Fukushima and Y. Hidaka, Resummation for the field-theoretical derivation of the negative magnetoresistance, JHEP 04 (2020) 162 [arXiv: 1906.02683] [INSPIRE].

[31] D.T. Son and N. Yamamoto, Berry curvature, triangle anomalies, and the chiral magnetic effect in Fermi liquids, Phys. Rev. Lett. 109 (2012) 181602 [arXiv:1203.2697] [INSPIRE].

[32] D.T. Son and N. Yamamoto, Kinetic theory with Berry curvature from quantum field theories, Phys. Rev. D 87 (2013) 085016 [arXiv:1210.8158] [inSPIRE].

[33] M.A. Stephanov and Y. Yin, Chiral kinetic theory, Phys. Rev. Lett. 109 (2012) 162001 [arXiv: 1207.0747] [INSPIRE].

[34] J.-H. Gao, Z.-T. Liang, S. Pu, Q. Wang and X.-N. Wang, Chiral anomaly and local polarization effect from quantum kinetic approach, Phys. Rev. Lett. 109 (2012) 232301 [arXiv: 1203.0725] [INSPIRE].

[35] S. $\mathrm{Pu}$, J.-H. Gao and Q. Wang, A consistent description of kinetic equation with triangle anomaly, Phys. Rev. D 83 (2011) 094017 [arXiv: 1008.2418] [INSPIRE].

[36] J.-W. Chen, S. Pu, Q. Wang and X.-N. Wang, Berry curvature and four-dimensional monopoles in the relativistic chiral kinetic equation, Phys. Rev. Lett. 110 (2013) 262301 [arXiv: 1210.8312] [INSPIRE]. 
[37] Y. Hidaka, S. Pu and D.-L. Yang, Relativistic chiral kinetic theory from quantum field theories, Phys. Rev. D 95 (2017) 091901 [arXiv: 1612.04630] [INSPIRE].

[38] C. Manuel and J.M. Torres-Rincon, Kinetic theory of chiral relativistic plasmas and energy density of their gauge collective excitations, Phys. Rev. D 89 (2014) 096002 [arXiv: 1312.1158] [INSPIRE].

[39] C. Manuel and J.M. Torres-Rincon, Chiral transport equation from the quantum Dirac Hamiltonian and the on-shell effective field theory, Phys. Rev. D 90 (2014) 076007 [arXiv: 1404.6409] [INSPIRE].

[40] Y. Wu, D. Hou and H.-C. Ren, Field theoretic perspectives of the Wigner function formulation of the chiral magnetic effect, Phys. Rev. D 96 (2017) 096015 [arXiv: 1601.06520] [INSPIRE].

[41] N. Mueller and R. Venugopalan, Worldline construction of a covariant chiral kinetic theory, Phys. Rev. D 96 (2017) 016023 [arXiv:1702.01233] [inSPIRE].

[42] N. Mueller and R. Venugopalan, The chiral anomaly, Berry's phase and chiral kinetic theory, from world-lines in quantum field theory, Phys. Rev. D 97 (2018) 051901 [arXiv: 1701.03331] [INSPIRE].

[43] A. Huang, S. Shi, Y. Jiang, J. Liao and P. Zhuang, Complete and consistent chiral transport from Wigner function formalism, Phys. Rev. D 98 (2018) 036010 [arXiv:1801.03640] [INSPIRE].

[44] J.-H. Gao, Z.-T. Liang, Q. Wang and X.-N. Wang, Disentangling covariant Wigner functions for chiral fermions, Phys. Rev. D 98 (2018) 036019 [arXiv: 1802.06216] [INSPIRE].

[45] S. Carignano, C. Manuel and J.M. Torres-Rincon, Consistent relativistic chiral kinetic theory: a derivation from on-shell effective field theory, Phys. Rev. D 98 (2018) 076005 [arXiv: 1806.01684] [INSPIRE].

[46] S. Lin and A. Shukla, Chiral kinetic theory from effective field theory revisited, JHEP 06 (2019) 060 [arXiv: 1901.01528] [inSPIRE].

[47] S. Carignano, C. Manuel and J.M. Torres-Rincon, Chiral kinetic theory from the on-shell effective field theory: derivation of collision terms, Phys. Rev. D 102 (2020) 016003 [arXiv: 1908.00561] [INSPIRE].

[48] Y.-C. Liu, L.-L. Gao, K. Mameda and X.-G. Huang, Chiral kinetic theory in curved spacetime, Phys. Rev. D 99 (2019) 085014 [arXiv:1812.10127] [INSPIRE].

[49] N. Weickgenannt, X.-L. Sheng, E. Speranza, Q. Wang and D.H. Rischke, Kinetic theory for massive spin-1/2 particles from the Wigner-function formalism, Phys. Rev. D 100 (2019) 056018 [arXiv: 1902 .06513] [INSPIRE].

[50] J.-H. Gao and Z.-T. Liang, Relativistic quantum kinetic theory for massive fermions and spin effects, Phys. Rev. D 100 (2019) 056021 [arXiv: 1902.06510] [INSPIRE].

[51] K. Hattori, Y. Hidaka and D.-L. Yang, Axial kinetic theory and spin transport for fermions with arbitrary mass, Phys. Rev. D 100 (2019) 096011 [arXiv:1903.01653] [INSPIRE].

[52] Z. Wang, X. Guo, S. Shi and P. Zhuang, Mass correction to chiral kinetic equations, Phys. Rev. D 100 (2019) 014015 [arXiv: 1903.03461] [InSPIRE].

[53] D.-L. Yang, K. Hattori and Y. Hidaka, Effective quantum kinetic theory for spin transport of fermions with collsional effects, JHEP 07 (2020) 070 [arXiv:2002.02612] [INSPIRE]. 
[54] Y.-C. Liu, K. Mameda and X.-G. Huang, Covariant spin kinetic theory I: collisionless limit, Chin. Phys. C 44 (2020) 094101 [arXiv: 2002. 03753] [INSPIRE].

[55] T. Hayata, Y. Hidaka and K. Mameda, Second order chiral kinetic theory under gravity and antiparallel charge-energy flow, JHEP 05 (2021) 023 [arXiv: 2012.12494] [INSPIRE].

[56] S. Chen, Z. Wang and P. Zhuang, Equal-time kinetic equations in a rotational field, arXiv:2101.07596 [INSPIRE].

[57] D. Vasak, M. Gyulassy and H.T. Elze, Quantum transport theory for Abelian plasmas, Annals Phys. 173 (1987) 462 [inSPIRE].

[58] H.T. Elze, M. Gyulassy and D. Vasak, Transport equations for the QCD quark Wigner operator, Nucl. Phys. B 276 (1986) 706 [INSPIRE].

[59] H.-T. Elze and U.W. Heinz, Quark-gluon transport theory, Phys. Rept. 183 (1989) 81 [INSPIRE].

[60] P. Zhuang and U.W. Heinz, Relativistic quantum transport theory for electrodynamics, Annals Phys. 245 (1996) 311 [nucl-th/9502034] [INSPIRE].

[61] X.-L. Sheng, Wigner function for spin-1/2 fermions in electromagnetic fields, Ph.D. thesis, Frankfurt U., Frankfurt, Germany (2019) [arXiv:1912.01169] [INSPIRE].

[62] J.-Y. Chen, D.T. Son and M.A. Stephanov, Collisions in chiral kinetic theory, Phys. Rev. Lett. 115 (2015) 021601 [arXiv: 1502.06966] [INSPIRE].

[63] J.-H. Gao, J.-Y. Pang and Q. Wang, Chiral vortical effect in Wigner function approach, Phys. Rev. D 100 (2019) 016008 [arXiv: 1810.02028] [InSPIRE].

[64] P. Kovtun, Lectures on hydrodynamic fluctuations in relativistic theories, J. Phys. A 45 (2012) 473001 [arXiv: 1205.5040] [INSPIRE].

[65] S.-Z. Yang, J.-H. Gao, Z.-T. Liang and Q. Wang, Second-order charge currents and stress tensor in a chiral system, Phys. Rev. D 102 (2020) 116024 [arXiv: 2003.04517] [INSPIRE].

[66] N. Weickgenannt, E. Speranza, X.-L. Sheng, Q. Wang and D.H. Rischke, Generating spin polarization from vorticity through nonlocal collisions, arXiv:2005.01506 [INSPIRE].

[67] Z. Wang, X. Guo and P. Zhuang, Local equilibrium spin distribution from detailed balance, arXiv:2009.10930 [INSPIRE].

[68] N. Weickgenannt, E. Speranza, X.-L. Sheng, Q. Wang and D.H. Rischke, Derivation of the nonlocal collision term in the relativistic Boltzmann equation for massive spin-1/2 particles from quantum field theory, arXiv:2103.04896 [INSPIRE].

[69] X.-L. Sheng, N. Weickgenannt, E. Speranza, D.H. Rischke and Q. Wang, From Kadanoff-Baym to Boltzmann equations for massive spin-1/2 fermions, arXiv:2103.10636 [INSPIRE].

[70] D. Hou and S. Lin, Polarization rotation of chiral fermions in vortical fluid, Phys. Lett. B 818 (2021) 136386 [arXiv: 2008.03862] [INSPIRE]. 